\title{
Beyond Talagrand Functions: New Lower Bounds for Testing Monotonicity and Unateness*
}

\author{
Xi Chen \\ Columbia University \\ New York, NY, USA 10027 \\ xichen@cs.columbia.edu
}

\author{
Erik Waingarten \\ Columbia University \\ New York, NY, USA 10027 \\ eaw@cs.columbia.edu
}

\author{
Jinyu Xie \\ Columbia University \\ New York, NY, USA 10027 \\ jinyu@cs.columbia.edu
}

\begin{abstract}
We prove a lower bound of $\widetilde{\Omega}\left(n^{1 / 3}\right)$ for the query complexity of any two-sided and adaptive algorithm that tests whether an unknown Boolean function $f:\{0,1\}^{n} \rightarrow\{0,1\}$ is monotone versus far from monotone. This improves the recent lower bound of $\widetilde{\Omega}\left(n^{1 / 4}\right)$ for the same problem by Belovs and Blais (STOC'16). Our result builds on a new family of random Boolean functions that can be viewed as a two-level extension of Talagrand's random DNFs.

Beyond monotonicity we prove a lower bound of $\widetilde{\Omega}(\sqrt{n})$ for twosided, adaptive algorithms and a lower bound of $\widetilde{\Omega}(n)$ for one-sided, non-adaptive algorithms for testing unateness, a natural generalization of monotonicity. The latter matches the linear upper bounds by Khot and Shinkar (RANDOM'16) and by Baleshzar, Chakrabarty, Pallavoor, Raskhodnikova, and Seshadhri (2017).
\end{abstract}

\section{CCS CONCEPTS}

- Theory of computation $\rightarrow$ Lower bounds and information complexity;

\section{KEYWORDS}

Property testing, monotonicity, unateness, Boolean functions

ACM Reference format:

Xi Chen, Erik Waingarten, and Jinyu Xie. 2017. Beyond Talagrand Functions: New Lower Bounds for Testing Monotonicity and Unateness. In Proceedings of 49th Annual ACM SIGACT Symposium on the Theory of Computing, Montreal, Canada, June 2017 (STOC'17), 14 pages.

DOI: $10.1145 / 3055399.3055461$

\section{INTRODUCTION}

Over the last few decades, property testing has emerged as an important line of research in sublinear time algorithms. The goal is to understand abilities and limitations of randomized algorithms that determine whether an unknown object has a specific property or is far from having the property, by examining randomly a small portion of the object. Over the years many different types of objects

*The full version of the paper is available at [16].

Permission to make digital or hard copies of all or part of this work for personal or classroom use is granted without fee provided that copies are not made or distributed for profit or commercial advantage and that copies bear this notice and the full citation on the first page. Copyrights for components of this work owned by others than ACM must be honored. Abstracting with credit is permitted. To copy otherwise, or republish, to post on servers or to redistribute to lists, requires prior specific permission and/or a fee. Request permissions from permissions@acm.org.

STOC'17, Montreal, Canada

(C) 2017 ACM. 978-1-4503-4528-6/17/06 ..\$15.00

DOI: $10.1145 / 3055399.3055461$ and properties have been studied from this property testing perspective (see $[21,28,29]$ for overviews of contemporary property testing research).

In this paper we study the monotonicity testing of Boolean functions, one of the most basic and natural problems that have been studied in the area of property testing for many years $[1,4,5,7-$ $12,14,15,17-20,23-25,30,31]$, with many exciting developments during the past few years. Introduced by Goldreich, Goldwasser, Lehman, and Ron [22], the problem is concerned with the (randomized) query complexity of determining whether an unknown Boolean function $f:\{0,1\}^{n} \rightarrow\{0,1\}$ is monotone or far from monotone. Recall that $f$ is monotone if $f(x) \leq f(y)$ for all $x<y$ (i.e., $x_{i} \leq y_{i}$ for every $\left.i \in[n]=\{1, \ldots, n\}\right)$. We say that $f$ is $\varepsilon$-close to monotone if $\operatorname{Pr}[f(\boldsymbol{x}) \neq g(\boldsymbol{x})] \leq \varepsilon$ for some monotone function $g$, where the probability is taken over a uniform draw of $\boldsymbol{x}$ from $\{0,1\}^{n}$, and that $f$ is $\varepsilon$-far from monotone otherwise.

We are interested in query-efficient randomized algorithms for the following task: Given as input a distance parameter $\varepsilon>0$ and oracle access to an unknown Boolean function $f:\{0,1\}^{n} \rightarrow\{0,1\}$, accept with probability at least $2 / 3$ if $f$ is monotone and reject with probability at least $2 / 3$ if $f$ is $\varepsilon$-far from monotone.

Beyond monotonicity, we also work on the testing of unateness, a generalization of monotonicity. Here a Boolean function $f:\{0,1\}^{n} \rightarrow\{0,1\}$ is unate if there exists a string $r \in\{0,1\}^{n}$ such that $g(x)=f(x \oplus r)$ is monotone (i.e., $f$ is either nondecreasing or nonincreasing in each coordinate), where we use $\oplus$ to denote the bitwise XOR of two strings. We are interested in query-efficient randomized algorithms that determine whether an unknown $f$ is unate or far from unate.

\subsection{Previous Work on Monotonicity Testing and Unateness Testing}

The work of Goldreich et al. [22, 23] proposed a simple "edge tester." For each round, the "edge tester" picks an $x \in\{0,1\}^{n}$ and an $i \in[n]$ uniformly at random and queries both $f(x)$ and $f(y)$ with $y=x^{(i)}$, where $x^{(i)}$ denotes $x$ with its $i$ th bit flipped. If $(x, y)$ is a violating edge, i.e., either 1) $x<y$ and $f(x)>f(y)$ or 2) $y<x$ and $f(y)>$ $f(x)$, the tester rejects $f$; the tester accepts $f$ if no violating edge is found after a certain number of rounds.

The "edge tester" is both one-sided (i.e. it always accept when $f$ is monotone) and non-adaptive (i.e.its queries do not depend on the oracle's responses to previous queries). [23] showed that $O(n / \varepsilon)$ rounds suffice for the "edge tester" to find a violating edge with high probability when $f$ is $\varepsilon$-far from monotone.

Later Fischer et al. [20] obtained the first lower bounds, showing that there is a constant distance parameter $\varepsilon_{0}>0$ such that $\Omega(\log n)$ 
queries are necessary for any non-adaptive algorithm and $\Omega(\sqrt{n})$ queries are necessary for any non-adaptive, one-sided algorithm.

These were the best known results for more than a decade, until Chakrabarty and Seshadhri improved the linear upper bound of Goldreich et al. to $\widetilde{O}\left(n^{7 / 8} \varepsilon^{-3 / 2}\right)$ [10] using a "pair tester" which is one-sided and non-adaptive. Such a tester looks for a so-called violating pair $(x, y)$ of $f$ satisfying $x<y$ and $f(x)>f(y)$. Their analysis was later slightly refined by Chen et al. in [15] to $\widetilde{O}\left(n^{5 / 6} \varepsilon^{-4}\right)$. [15] also gave an $\widetilde{\Omega}\left(n^{1 / 5}\right)$ lower bound for non-adaptive algorithms.

Further progress has been made during the past two years. Chen et al. [14] obtained a lower bound of $\Omega\left(n^{1 / 2-c}\right)$ for non-adaptive algorithms, for any positive constant $c$. Later an upper bound of $\widetilde{O}\left(n^{1 / 2} / \epsilon^{2}\right)$ was obtained by Khot et al. [25] via a deep analysis of the "pair tester" based on a new isoperimetric-type theorem for far-from-monotone Boolean functions. These results (almost) resolved the query complexity of non-adaptive monotonicity testing over Boolean functions. Very recently Belovs and Blais [5] made a breakthrough and gave an $\widetilde{\Omega}\left(n^{1 / 4}\right)$ lower bound for adaptive algorithms. This is the first polynomial lower bound for adaptive monotonicity testing. We discuss the lower bound construction of [5] in more detail in Section 1.3.

The problem of testing unateness was introduced in the same paper [23] by Goldreich et al. where they obtained a one-sided and non-adaptive algorithm with $O\left(n^{3 / 2} / \varepsilon\right)$ queries. The first improvement after [23] was made by Khot and Shinkar [26] with a one-sided and adaptive $O(n \log n / \varepsilon)$-query algorithm. Baleshzar et al. [3] extended the algorithm of [26] to testing unateness of functions $f$ : $\{0,1\}^{n} \rightarrow \mathbb{R}$ with the same query complexity. They also gave a lower bound of $\Omega(\sqrt{n} / \varepsilon)$ for one-sided and non-adaptive algorithms over Boolean functions. Baleshzar et al. [2,13] recently gave a one-sided and non-adaptive algorithm of $O((n / \varepsilon) \log (n / \varepsilon))$ queries, as well as a one-sided and adaptive algorithm of $O(n / \varepsilon)$ queries.

\subsection{Our Results}

The main result of this work is an $\widetilde{\Omega}\left(n^{1 / 3}\right)$ query lower bound for adaptive monotonicity testing of Boolean functions, improving the $\widetilde{\Omega}\left(n^{1 / 4}\right)$ lower bound of Belovs and Blais [5].

Theorem 1.1 (Monotonicity). There exists a constant $\varepsilon_{0}>0$ such that any two-sided and adaptive algorithm for testing whether an unknown Boolean function $f:\{0,1\}^{n} \rightarrow\{0,1\}$ is monotone or $\varepsilon_{0}$-far from monotone must make $\Omega\left(n^{1 / 3} / \log ^{2} n\right)$ queries.

In [5] Belovs and Blais obtained their $\widetilde{\Omega}\left(n^{1 / 4}\right)$ lower bound using a family of random functions known as Talagrand's random DNFs (or simply as the Talagrand function) [32]. A function drawn from this family is the disjunction of $N \equiv 2^{\sqrt{n}}$ many monotone terms $T_{i}$ with each $T_{i}$ being the conjunction of $\sqrt{n}$ variables sampled uniformly from $[n]$. So such a function looks like

$$
f(x)=\bigvee_{i \in[N]} T_{i}(x)=\bigvee_{i \in[N]}\left(\bigwedge_{k \in S_{i}} x_{k}\right) .
$$

However, it turns out that there is a matching $\widetilde{O}\left(n^{1 / 4}\right)$-query, onesided algorithm for functions of [5]. (See Section 4 for a sketch of the algorithm.) So the analysis of [5] is tight.

Our main contribution behind the lower bound of Theorem 1.1 is a new and harder family of random functions for monotonicity testing, which we call two-level Talagrand functions. This starts by re-examining the construction of [5] from a slightly different angle, which leads to both natural generalizations and simpler analysis of such functions.

We review the construction of [5] under this framework and describe our new two-level Talagrand functions in Section 1.3. As far as we know, we are not aware of the two-level Talagrand functions in the literature and expect to see more interesting applications of them in the future. On the other hand, the techniques developed in the proof of Theorem 1.1 can be easily adapted to obtain a tight $\widetilde{\Omega}\left(n^{1 / 2}\right)$ lower bound for non-adaptive monotonicity testing, removing the $-c$ in $\Omega\left(n^{1 / 2-c}\right)$ lower bound of [14].

Theorem 1.2 (Non-ADAPTIVE MONOTONICITY). There exists a constant $\varepsilon_{0}>0$ such that any two-sided, non-adaptive algorithm for testing whether an unknown Boolean function $f:\{0,1\}^{n} \rightarrow\{0,1\}$ is monotone or $\varepsilon_{0}$-far from monotone must make $\widetilde{\Omega}\left(n^{1 / 2}\right)$ queries.

Next for testing unateness, we present an $\widetilde{\Omega}\left(n^{1 / 2}\right)$ lower bound against adaptive algorithms.

Theorem 1.3 (UnATENESs). There exists a constant $\varepsilon_{0}>0$ such that any two-sided and adaptive algorithm for testing whether an unknown Boolean function $f:\{0,1\}^{n} \rightarrow\{0,1\}$ is unate versus $\varepsilon_{0}$-far from unate must make $\Omega\left(n^{1 / 2} / \log ^{2} n\right)$ queries.

The lower bound construction behind Theorem 1.3 follows a similar framework. Some of the new ideas and techniques developed for the monotonicity lower bound are adapted to prove Theorem 1.3 though with a few twists that are unique to unateness.

Moreover, we obtain a linear lower bound for one-sided and nonadaptive unateness algorithms. This improves the $\Omega(\sqrt{n})$ lower bound of Baleshzar et al. [3], and matches the recent linear upper bounds of Baleshzar et al. [2,13] for such algorithms.

THEOREM 1.4 (ONE-SIDED AND NON-ADAPTIVE UNATENESS). There exists a constant $\varepsilon_{0}>0$ such that any one-sided, non-adaptive algorithm for testing whether an unknown Boolean function is unate versus $\varepsilon_{0}$-far from unate must make $\Omega\left(n / \log ^{2} n\right)$ queries.

We summarize previous work and our new results in Figure 1.

\subsection{An Overview of Our Construction for Theorem 1.1}

We start by reviewing the hard functions used in [5] (i.e., Talagrand's random DNFs), but this time interpret them under the new framework that we will follow in this paper. Employing Yao's minimax principle, the goal of [5] is to (1) construct a pair of distributions $\mathcal{D}_{\text {yes }}^{*}$ and $\mathcal{D}_{\text {no }}^{*}$ over Boolean functions from $\{0,1\}^{n}$ to $\{0,1\}$ such that $f \sim \mathcal{D}_{\text {yes }}^{*}$ is always monotone while $\boldsymbol{g} \sim \mathcal{D}_{\text {no }}^{*}$ is $\Omega(1)$-far from monotone with probability $\Omega(1)$; (2) show that no deterministic algorithm with a small number of queries can distinguish them.

Let $N=2^{\sqrt{n}}$. A function $f$ is drawn from the distribution $\mathcal{D}_{\text {yes }}^{*}$ using the following procedure. We first sample a sequence of $N$ random sub-hypercubes $H_{i}$ in $\{0,1\}^{n}$. Each $H_{i}$ is defined by a random term $T_{i}$ with $x \in H_{i}$ if $T_{i}(x)=1$, where $T_{i}$ is the conjunction of $\sqrt{n}$ random variables sampled uniformly from $[n]$ (so each $H_{i}$ has dimension at most $n-\sqrt{n}$ ). By a simple calculation most likely the $H_{i}$ 's have little overlap between each other and they together cover 


\begin{tabular}{|c|c|c|c|}
\hline & Best Upper Bound & Best Lower Bound & This Work \\
\hline \hline Non-adaptive & & & \\
\hline \hline Monotonicity & $\widetilde{O}\left(\sqrt{n} / \varepsilon^{2}\right)[25]$ & $\widetilde{\Omega}\left(n^{1 / 2-c}\right)[14]$ & $\widetilde{\Omega}(\sqrt{n})$ \\
Unateness & $\widetilde{O}(n / \varepsilon)[13]$ & $\Omega(\sqrt{n})$ (one-sided) $[3]$ & $\widetilde{\Omega}(n)$ (one-sided) \\
\hline \hline Adaptive & & & \\
\hline \hline Monotonicity & $\widetilde{O}\left(\sqrt{n} / \varepsilon^{2}\right)[25]$ & $\widetilde{\Omega}\left(n^{1 / 4}\right)[5]$ & $\widetilde{\Omega}\left(n^{1 / 3}\right)$ \\
Unateness & $O(n / \varepsilon)[2,13,26]$ & & $\widetilde{\Omega}(\sqrt{n})$ \\
\hline
\end{tabular}

Figure 1: Previous work and our results on monotonicity testing and unateness testing.

an $\Omega(1)$-fraction of $\{0,1\}^{n}$. Informally we consider $H_{i}$ 's together as a random partition of $\{0,1\}^{n}$ where each $x \in\{0,1\}^{n}$ belongs to a unique $H_{i}$ (for now do not worry about cases when $x$ lies in none or multiple $H_{i}$ 's). Next we sample for each $H_{i}$ a random dictatorship function $h_{i}(x)=x_{\ell}$ with $\ell$ drawn uniformly from $[n]$. The final $f$ is $f(x)=h_{i}(x)$ for each $x \in H_{i}$ (again do not worry about cases when $x$ lies in none or multiple $H_{i}$ 's). A function $g$ from $\mathcal{D}_{\text {no }}^{*}$ is drawn using the same procedure except that each $h_{i}$ is a random anti-dictatorship function $h_{i}(x)=\overline{x_{\ell}}$ with $\ell$ sampled from $[n]$.

Note that the distributions sketched here are slightly different from [5] (see Section 4). For $\mathcal{D}_{\text {no }}^{*}$ in particular, instead of associating each $H_{i}$ with an independent, random anti-dictatorship $h_{i}$, [5] draws $\sqrt{n}$ anti-dictatorship functions in total and then associates each $H_{i}$ with one of them randomly. ${ }^{1}$ While this gives a connection to the noise sensitivity results of [27] on Talagrand functions, it makes the functions harder to analyze and generalize due to the correlation between $h_{i}$ 's.

By definition, $f$ is always monotone. On the other hand, $g$ is far from monotone as (intuitively) $H_{i}$ 's are mostly disjoint and within each $H_{i}, g$ is anti-monotone due to the anti-dictatorship $h_{i}$.

At a high level one can view the $T_{i}$ 's together as an addressing function that maps each string $x$ to one of the $N$ independent antidictatorship functions $h_{i}$, by randomly partitioning $\{0,1\}^{n}$ using a long sequence of very small hypercubes $H_{i}$.

Conceptually, this is the picture that we will follow to define our two-level Talagrand functions. They will also be built using a random partition of $\{0,1\}^{n}$ into a sequence of small(er) hypercubes, with the property that (i) if one places a dictatorship function in each hypercube independently at random, the resulting function is monotone, and (ii) if one places a random anti-dictatorship function in each of them, the resulting function is far from monotone with $\Omega(1)$ probability. The main difference lies in the way how the partition is done and how the hypercubes are sampled.

Before introducing the two-level Talagrand function, we explain at a high-level why the pair of distributions $\mathcal{D}_{\text {yes }}^{*}$ and $\mathcal{D}_{\text {no }}^{*}$ are hard to distinguish (this will allow us to compare them with our new functions and see why the latter are harder). Consider the situation when an algorithm is given an $x \in H_{i}$ 's with $h_{i}(x)=0$ and would like to find a violating pair in $H_{i}$, by flipping some 1's of $x$ to 0 and hoping to see $g(y)=1$ in the new $y$ obtained. The algorithm faces the following dilemma:

\footnotetext{
${ }^{1}$ This is close but also not exactly the same as the distributions of [5]; see Section 4 .
}

(1) on the one hand, the algorithm wants to flip as many 1's of $x$ as possible in order to flip the hidden anti-dictator variable $\ell$ of the anti-dictatorship function $h_{i}$;

(2) on the other hand, it is very unlikely for the algorithm to flip many (say $\omega(\sqrt{n} \log n))$ 's of $x$ without moving $y$ outside of $H_{i}$ (which happens if one of the 1-entries flipped lies in $T_{i}$ ), and when this happens, $g(y)$ provides essentially no information about $\ell$.

So $g$ is very resilient against such attacks. However, consider the case when $x \in H_{i}$ and $h_{i}(x)=1$; then, the algorithm may try to find a violating pair in $H_{i}$ by flipping many 0 's of $x$ to 1 , and this time there is no limitation on how many 0's of $x$ one can flip! In fact flipping 0's to 1's can never move $y$ outside of $H_{i}{ }^{2}$ In Section 4 we leverage this observation to find a violation with $\widetilde{O}\left(n^{1 / 4}\right)$ queries.

Now we describe the two-level Talagrand function. The random partitions we employ below are more complex; they allow us to upperbound not only the number of 1's of $x$ that an algorithm can flip (without moving outside of the hypercube) but also the number of 0 's as well. We use $\mathcal{D}_{\text {yes }}$ and $\mathcal{D}_{\text {no }}$ to denote the two distributions.

To draw a function $f$ from $\mathcal{D}_{\text {yes }}$, we partition $\{0,1\}^{n}$ into $N^{2}$ random sub-hypercubes as follows. First we sample as before $N$ random $\sqrt{n}$-terms $T_{i}$ to obtain $H_{i}$. After that, we further partition each $H_{i}$, by independently sampling $N$ random $\sqrt{n}$-clauses $C_{i, j}$, with each of them being the disjunction of $\sqrt{n}$ random variables sampled from $[n]$ uniformly. The terms $T_{i}$ and clauses $C_{i, j}$ together define $N^{2}$ sub-hypercubes $H_{i, j}: x \in H_{i, j}$ if $T_{i}(x)=1$ and $C_{i, j}(x)=0$. The rest is very similar. We sample a random dictatorship function $h_{i, j}$ for each $H_{i, j}$, and the function $f$ has $f(x)=h_{i, j}(x)$ for $x \in H_{i, j}{ }^{3}$ A function $g$ from $\mathcal{D}_{\text {no }}$ is drawn using the same procedure except that $h_{i, j}$ 's are independent random anti-dictatorship functions.

We call such functions two-level Talagrand functions, as one can view each of them as a two-level structure with the top being a Talagrand DNF and the bottom being $N$ Talagrand CNFs, one attached with each term of the top DNF. See Figure 3 for a visual depiction of such functions, with the formal definition of $\mathcal{D}_{\text {yes }}$ and $\mathcal{D}_{\text {no }}$ presented in Section 3.1.

\footnotetext{
${ }^{2}$ While we tried to keep the high-level description in this part simple, there is indeed a truncation that is always done on $g$, where one set $g(x)=1$ for $|x|>(n / 2)+\sqrt{n}$, $g(x)=0$ for $|x|<(n / 2)-\sqrt{n}$, and keep $g(x)$ the same when $x$ lies in the middle layers with $|x|$ between $(n / 2)-\sqrt{n}$ and $(n / 2)+\sqrt{n}$. But even with the truncation in place, one can take advantage of this observation and find a violation in $g$ using only $\widetilde{O}\left(n^{1 / 4}\right)$ queries. See details in Section 4

${ }^{3}$ Again, do not worry about cases when $x$ lies in none or multiple $H_{i, j}$ 's.
} 
By a simple calculation, (most likely) the $H_{i, j}$ 's have very little overlap and cover an $\Omega(1)$-fraction of $\{0,1\}^{n}$. This is why $g$ is far from monotone.

Conceptually, the definition of $\mathcal{D}_{\text {yes }}$ and $\mathcal{D}_{\text {no }}$ follows the same high-level picture: the terms $T_{i}$ and clauses $C_{i, j}$ together serve as an addressing function, which we refer to as a multiplexer in the proof (see Figure 2 for a visual depiction). It maps each string $x$ to one of the $N^{2}$ independent and random dictatorship or anti-dictatorship $h_{i^{*}, j^{*}}$. Terms $T_{i}$ in the first level of multiplexing determines $i^{*}$ and clauses $C_{i^{*}, j}$ in the second level of multiplexing determines $j^{*}$.

The two-level Talagrand functions are harder than those used in [5] since, starting with an $x \in H_{i, j}$, not only flipping $\omega(\sqrt{n} \log n)$ many 1's would move it outside of $H_{i, j}$ with high probability (because the term $T_{i}$ is most likely no longer satisfied), the same holds when flipping $\omega(\sqrt{n} \log n)$ many 0 's to 1 (because the clause $C_{i, j}$ is most likely no longer falsified).

\subsection{An Overview of the Proof of Theorem 1.1}

Let $q=n^{1 / 3} / \log ^{2} n$ and let $B$ be a $q$-query deterministic algorithm, which we view equivalently as a binary decision tree of depth $q$. Our goal is to prove the following for $\mathcal{D}_{\text {yes }}$ and $\mathcal{D}_{\text {no: }}$ :

$$
\underset{f \sim \mathcal{D}_{\text {yes }}}{\operatorname{Pr}}[B \text { accepts } f] \leq \underset{\boldsymbol{g} \sim \mathcal{D}_{\text {no }}}{\operatorname{Pr}}[B \text { accepts } g]+o(1) .
$$

To prove (1), it suffices to show for every leaf $\ell$ of $B$,

$$
\underset{f \sim \mathcal{D}_{\text {yes }}}{\operatorname{Pr}}[f \text { reaches } \ell] \leq(1+o(1)) \cdot \underset{\boldsymbol{g} \sim \mathcal{D}_{\text {no }}}{\operatorname{Pr}}[\boldsymbol{g} \text { reaches } \ell] .
$$

However, this is challenging because both events above are highly complex. Following the same idea used in [6], we decompose such events into simpler events by allowing the oracle to return more than just $f(x)$. Upon each query $x \in\{0,1\}^{n}$, the oracle returns the so-called signature of $x$. When $x$ satisfies a unique term $T_{i^{*}}$, the signature reveals the index $i^{*}$. The same happens to the second level: when $x$ falsifies a unique clause $C_{i^{*}}, j^{*}$, the signature reveals the index $j^{*}$. (See the formal definition for what happens when $x$ satisfies, or falsifies, none or multiple terms, or clauses.)

We consider deterministic $q$-query algorithms $B$ with access to this stronger oracle. We view $B$ as a decision tree in which each edge is labelled with a possible signature returned by the oracle. Hence the number of children of each internal node is huge. We refer to such a tree as a signature tree. Our new goal is to prove that every leaf $\ell$ of $B$ satisfies (2). However, this is not true in general. Instead we divide the leaves into good ones and bad ones, prove (2) for each good leaf and show that $f \sim \mathcal{D}_{\text {yes }}$ reaches a bad leaf with probability $o(1)$.

The definition of bad leaves and the proof of $f \sim \mathcal{D}_{\text {yes }}$ reaching one with $o(1)$ probability poses the main technical challenge. First, we characterize four types of edges where a bad event occurs and refer to them as bad edges; a leaf $\ell$ then is bad if the root-to- $\ell$ path has a bad edge. These bad edges help us rule out certain attacks an algorithm may try. The first two events formalize the notion we highlighted earlier that given a string $y$ queried before, flipping $\omega(\sqrt{n} \log n)$ many 1's of $y$ to 0 's, or 0 's to 1 's, results in a new string $x$ that most likely lies in a different sub-hypercube. The second two events formalize the notion that if queries do not flip many 1's to 0 's, or 0's to 1's, then observing a violating pair is unlikely.
In a bit more detail, the first two events are that (we let $A_{i, 1}$ and $A_{i, j, 0}$ denote the common 1-entries of strings queried so far that satisfy the same term $T_{i}$ and common 0 -entries of strings so far that falsify the same clause $C_{i, j}$, respectively) after a new query $x,\left|A_{i, 1}\right|$ or $\left|A_{i, j, 0}\right|$ drops by more than $\sqrt{n} \log n$. Such events occur when $x$ satisfies the same $T_{i}$ but has many 0 -entries in $A_{i, 1}$, or $x$ falsifies the same clause $C_{i, j}$ but has many 1 -entries in $A_{i, j, 0}$. Intuitively such events are unlikely to happen since before $x$ is queried, $T_{i}$ (or $C_{i, j}$ ) is "almost" 4 uniform over $A_{i, 1}$ (or $A_{i, j, 0}$ ). As a result, it is unlikely for the $\sqrt{n} \log n$ many 0 -entries of $x$ in $A_{i, 1}$ (1-entries of $x$ in $A_{i, j, 0}$ ) to entirely avoid $T_{i}\left(C_{i, j}\right)$. We follow this intuition to show that the probability of $f \sim \mathcal{D}_{\text {yes }}$ taking one such edge when walking down the tree is $o(1)$, which then allows us to prune such edges.

Organization. We introduce some notation and review the characterization of distance to monotonicity in Section 2 . We also prove two basic tree pruning lemmas. We prove the $\widetilde{\Omega}\left(n^{1 / 3}\right)$ lower bound (Theorem 1.1) for adaptive monotonicity testing in Section 3. We sketch in Section 4 an adaptive algorithm for testing monotonicity of the one-level Talagrand functions from [5] using $O\left(n^{1 / 4}\right)$ queries, and an adaptive algorithm for testing monotonicity of the two-level Talagrand functions using $O\left(n^{1 / 3}\right)$ queries. We conclude with discussion and open problems in Section 5. Proofs of Theorems 1.2, 1.3 , and 1.4 can be found in the full version [16].

\section{PRELIMINARIES}

We use bold font letters such as $T$ and $C$ for random variables. We write $[n]$ to denote $\{1, \ldots, n\}$. Given a string $x \in\{0,1\}^{n}$, we use $|x|$ to denote the number of 1's in $x$. Given an $x \in\{0,1\}^{n}$ and $S \subseteq[n]$, $x^{(S)}$ denotes the string obtained from $x$ by flipping each entry $x_{i}$ with $i \in S$. When $S=\{i\}$, we write $x^{(i)}$ instead of $x^{(\{i\})}$.

We let $N=2^{\sqrt{n}}$ throughout the paper. We let $e_{i}$, for each $i \in[N]$, denote the string in $\{0,1\}^{N}$ with its $k$ th entry being 0 if $k \neq i$ and 1 if $k=i$; we let $e_{i, i^{\prime}}, i<i^{\prime} \in[N]$, denote the string in $\{0,1, *\}^{N}$ with its $k$ th entry being (1) 0 if $k<i^{\prime}$ and $k \neq i$, (2) 1 if $k=i$ or $i^{\prime}$, and (3) $*$ if $k>i^{\prime}$. We let $\bar{e}_{i}$ (or $\bar{e}_{i, i^{\prime}}$ ) denote the string obtained from $e_{i}$ (or $e_{i, i^{\prime}}$ ) by flipping its 0 -entries to 1 and 1 -entries to 0 .

The distance $\operatorname{dist}(f, g)$ between two Boolean functions $f$ and $g$ is given as $\operatorname{Pr}_{\boldsymbol{x} \sim\{0,1\}^{n}}[f(\boldsymbol{x}) \neq g(\boldsymbol{x})]$ over the uniform distribution. The distance of $f$ to monotonicity, denoted by $\operatorname{dist}(f$, Mono $)$, is the minimum value of $\operatorname{dist}(f, g)$ over all monotone $g$.

We recall the following characterization (Lemma 4 in [20]):

Lemma 2.1. Let $f:\{0,1\}^{n} \rightarrow\{0,1\}$ be a Boolean function. Then

$$
\operatorname{dist}(f, M O N O)=|M| / 2^{n}
$$

where $M$ is a maximum set of disjoint violating pairs of $f$.

\subsection{Tree Pruning Lemmas}

We consider a rather general setup where a $q$-query deterministic algorithm $A$ has oracle access to an object $\boldsymbol{O}$ drawn from a distribution $\mathcal{D}$ : Upon each query $w$, the oracle with an object $O$ returns $\eta(w, O)$, an element from a finite set $\mathfrak{P}$. Such an algorithm can be equivalently viewed as a tree of depth $q$, where each internal node

\footnotetext{
${ }^{4}$ The distribution is not exactly uniform because we also need to consider strings that are known to not satisfy $T_{i}$ or not falsify $C_{i, j}$ as revealed in their signatures, though we will see in the proof that their influence is very minor.
} 


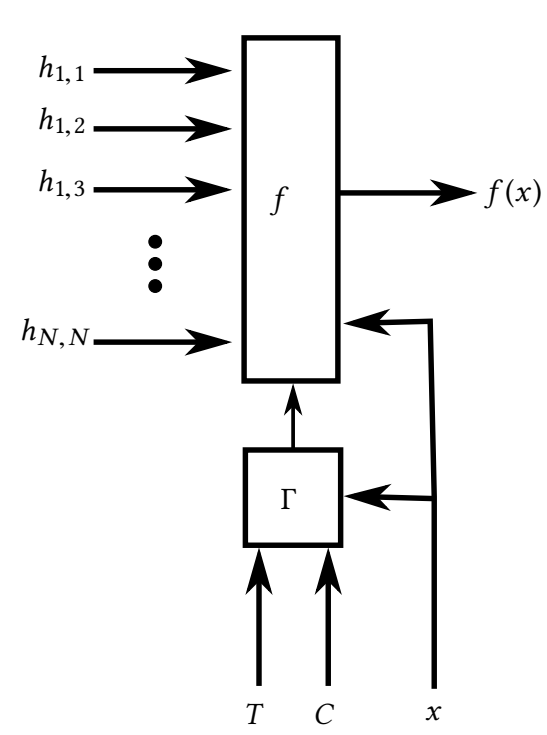

Figure 2: An illustration of the function $f=f_{T, C, H}$ from

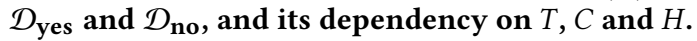

$u$ is labelled a query $w$ to make and has $|\mathfrak{P}|$ edges $(u, v)$ leaving $u$, each labelled a distinct element from $\mathfrak{P}$. (In general the degree of $u$ can be much larger than two; this is the case for all our applications later since we will introduce new oracles that upon a query $x \in\{0,1\}^{n}$ returns more information than $f(x)$.) In this section we do not care about labels of leaves of $A$, and present two basic pruning techniques that will be used in our analysis in Section 3.

Both lemmas share the following setup. Given $A$ and a set $E$ of edges of $A$ we use $L_{E}$ to denote the set of leaves $\ell$ that has at least one edge in $E$ along the path from the root to $\ell$. Each lemma below states that if $E$ satisfies certain properties with respect to $\mathcal{D}$ that we are interested in, then

$$
\underset{O \sim \mathcal{D}}{\operatorname{Pr}}\left[\boldsymbol{O} \text { reaches a leaf in } L_{E}\right]=o(1) .
$$

This will later allow us to focus on root-to-leaf paths that do not take any edge in $E$.

For each node $u$ of tree $A$, we use $\operatorname{Pr}[u]$ to denote the probability of $\boldsymbol{O} \sim \mathcal{D}$ reaching $u$. When $u$ is an internal node with $\operatorname{Pr}[u]>0$ we use $q(u)$ to denote the following conditional probability:

$$
q(u)=\underset{O \sim \mathcal{D}}{\operatorname{Pr}}[\boldsymbol{O} \text { follows an edge in } E \text { at } u \mid O \text { reaches } u] .
$$

We state the first pruning lemma; the proof is elementary and can be found in the full version [16].

Lemma 2.2. Given $E$, if $q(u)=o(1 / q)$ for every internal node $u$ with $\operatorname{Pr}[u]>0$, then (3) holds.

Next, for each leaf $\ell$ with $\operatorname{Pr}[\ell]>0$, letting $u_{1} u_{2} \cdots u_{k+1}=\ell$ be the root-to- $\ell$ path, we use $q^{*}(\ell)$ to denote $\sum_{i \in[k]} q\left(u_{i}\right)$. We state the second lemma below; the proof can also be found in [16].

Lemma 2.3. If every leaf $\ell$ of $A$ with $\operatorname{Pr}[\ell]>0$ satisfies $q^{*}(\ell)=$ $o(1)$, then (3) holds.

\section{MONOTONICITY LOWER BOUND}

\subsection{Distributions}

For a fixed $n>0$, we describe a pair of distributions $\mathcal{D}_{\text {yes }}$ and $\mathcal{D}_{\text {no }}$ supported on Boolean functions $f:\{0,1\}^{n} \rightarrow\{0,1\}$. We then show that every $f \sim \mathcal{D}_{\text {yes }}$ is monotone, and $f \sim \mathcal{D}_{\text {no }}$ is $\Omega(1)$-far from monotone with probability $\Omega(1)$. Recall that $N=2^{\sqrt{n}}$.

A function $f \sim \mathcal{D}_{\text {yes }}$ is drawn using the following procedure:

(1) Sample a pair $(T, C) \sim \mathcal{E}$ (which we describe next). The pair $(T, C)$ is then used to define a multiplexer map ${ }^{5}$

$$
\Gamma=\Gamma_{T, C}:\{0,1\}^{n} \rightarrow([N] \times[N]) \cup\left\{0^{*}, 1^{*}\right\} .
$$

(2) Sample $\boldsymbol{H}=\left(\boldsymbol{h}_{i, j}: i, j \in[N]\right)$ from a distribution $\mathcal{E}_{\text {yes }}$, where each $\boldsymbol{h}_{i, j}:\{0,1\}^{n} \rightarrow\{0,1\}$ is a random dictatorship function, i.e., $\boldsymbol{h}_{i, j}(x)=x_{k}$ with $k$ sampled independently for each $\boldsymbol{h}_{i, j}$ and uniformly at random from $[n]$.

(3) Finally, we define $f=f_{T, C, H}:\{0,1\}^{n} \rightarrow\{0,1\}$ :

(a) $f(x)=1$ if $|x|>(n / 2)+\sqrt{n}$;

(b) $f(x)=0$ if $|x|<(n / 2)-\sqrt{n}$;

(c) otherwise $((n / 2)-\sqrt{n} \leq|x| \leq(n / 2)+\sqrt{n})$, we have

$$
f(x)= \begin{cases}0 & \text { if } \Gamma(x)=0^{*} \\ 1 & \text { if } \Gamma(x)=1^{*} \\ h_{\Gamma(x)}(x) & \text { otherwise (i.e., } \Gamma(x) \in[N] \times[N])\end{cases}
$$

On the other hand a function $f=f_{T, C, H} \sim \mathcal{D}_{\text {no }}$ is drawn using the same procedure, with the only difference being that $\boldsymbol{H}=\left(\boldsymbol{h}_{i, j}\right.$ : $i, j \in[N]$ ) is drawn from $\mathcal{E}_{\text {no }}$ (instead of $\mathcal{E}_{\text {yes }}$ ): each $\boldsymbol{h}_{i, j}(x)=\overline{x_{k}}$ is a random anti-dictatorship function with $k$ drawn independently and uniformly from $[n]$.

REMARK 1. Given the same truncation done in both $\mathcal{D}_{\text {yes }}$ and $\mathcal{D}_{\text {no, }}$, it suffices to show a lower bound against algorithms that query strings in the middle layers only: $(n / 2)-\sqrt{n} \leq|x| \leq(n / 2)+\sqrt{n}$.

Next we describe the distribution $\mathcal{E}$ in details. $\mathcal{E}$ is uniform over all pairs $(T, C)$ of the following form: $T=\left(T_{i}: i \in[N]\right)$ with $T_{i}$ : $[\sqrt{n}] \rightarrow[n]$ and $C=\left(C_{i, j}: i, j \in[N]\right)$ with $C_{i, j}:[\sqrt{n}] \rightarrow[n]$. We call $T_{i}$ 's the terms and $C_{i, j}$ 's the clauses.

Equivalently, to draw a pair $(T, C) \sim \mathcal{E}$ :

- For each $i \in[N]$, sample a random term $T_{i}$ by sampling $T_{i}(k)$ independently and uniformly from $[n]$ for each $k \in[\sqrt{n}]$, with $T_{i}(k)$ viewed as the $k$ th variable of $T_{i}$.

- For each $i, j \in[N]$, sample a random clause $C_{i, j}$ by sampling $C_{i, j}(k)$ independently and uniformly from $[n]$ for each $k$, with $C_{i, j}(k)$ viewed as the $k$ th variable of $C_{i, j}$. Given a pair $(T, C)$, we interpret $T_{i}$ as a (DNF) term and abuse the notation to write

$$
T_{i}(x)=\bigwedge_{k \in[\sqrt{n}]} x_{T_{i}(k)}
$$

as a Boolean function over $n$ variables. We say $x$ satisfies $T_{i}$ when $T_{i}(x)=1$. We interpret each $C_{i, j}$ as a (CNF) clause and write

$$
C_{i, j}(x)=\bigvee_{k \in[\sqrt{n}]} x_{C_{i, j}(k)}
$$

as a Boolean function over $n$ variables. Similarly we say $x$ falsifies $C_{i, j}$ when $C_{i, j}(x)=0$.

${ }^{5}$ We use $0^{*}$ and $1^{*}$ to denote two special symbols (instead of the Kleene closure). 


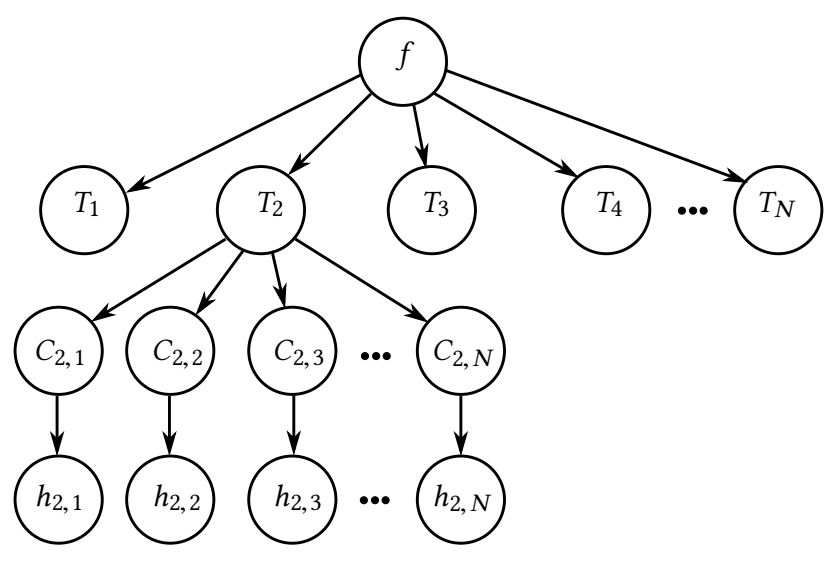

Figure 3: Picture of a function $f$ drawn from $\mathcal{D}_{\text {yes }}$ or $\mathcal{D}_{\text {no. }}$.

Each pair $(T, C)$ in the support of $\mathcal{E}$ defines a multiplexer map $\Gamma=\Gamma_{T, C}:\{0,1\}^{n} \rightarrow([N] \times[N]) \cup\left\{0^{*}, 1^{*}\right\}$. Informally speaking, $\Gamma$ consists of two levels: the first level uses the terms $T_{i}$ in $T$ to pick the first index $i^{\prime} \in[N]$; the second level uses the clauses $C_{i^{\prime}, j}$ in $C$ to pick the second index $j^{\prime} \in[N]$. Sometimes $\Gamma$ may choose to directly determine the value of the function by setting $\Gamma(x) \in\left\{0^{*}, 1^{*}\right\}$.

Formally, $(T, C)$ defines $\Gamma$ as follows. Given an $x \in\{0,1\}^{n}$ we have $\Gamma(x)=0^{*}$ if $T_{i}(x)=0$ for all $i \in[N]$ and $\Gamma(x)=1^{*}$ if $T_{i}(x)=1$ for at least two different $i$ 's in [N]. Otherwise there is a unique $i^{\prime}$ with $T_{i^{\prime}}(x)=1$, and the multiplexer enters the second level. Next, we have $\Gamma(x)=1^{*}$ if $C_{i^{\prime}, j}(x)=1$ for all $j \in[N]$ and $\Gamma(x)=0^{*}$ if $C_{i^{\prime}, j}(x)=0$ for at least two different $j^{\prime}$ 's in $[N]$. Otherwise there is a unique $j^{\prime} \in[N]$ with $C_{i^{\prime}, j^{\prime}}(x)=0$ and in this case the multiplexer outputs $\Gamma(x)=\left(i^{\prime}, j^{\prime}\right)$.

This finishes the definition of $\mathcal{D}_{\text {yes }}$ and $\mathcal{D}_{\text {no }}$. Figure 3 gives a graphical illustration of such functions. We now prove the prop-

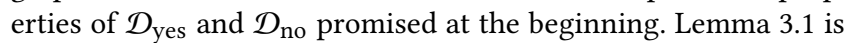
straightforward; its proof can be found in the full version [16].

Lemma 3.1. Every function $f$ in the support of $\mathcal{D}_{\text {yes }}$ is monotone.

Lemma 3.2. A function $f \sim \mathcal{D}_{\text {no }}$ is $\Omega(1)$-far-from monotone with probability $\Omega(1)$.

Proof. Fix a pair $(T, C)$ from the support of $\mathcal{E}$ and an $H$ from the support of $\mathcal{E}_{\text {no }}$. Let $f=f_{T, C, H}$.

Consider the set $X \subset\{0,1\}^{n}$ consisting of strings $x$ in the middle layers (i.e., $|x| \in(n / 2) \pm \sqrt{n})$ with $f(x)=1, \Gamma(x)=(i, j)$ for some $i, j$ $\in[N]$ (instead of $0^{*}$ or $1^{*}$ ), and $h_{i, j}$ being an anti-dictator function on the $k$ th variable for some $k \in[n]$ (so $x_{k}=0$ ). For each $x \in X$, we write $\eta(x)$ to denote the anti-dictator variable $k$ in $h_{i, j}$ and use $x^{*}$ to denote $x^{(\eta(x))}$. (Ideally, we would like to conclude that $\left(x, x^{*}\right)$ is a violating edge of $f$ as $h_{i, j}\left(x^{*}\right)=0$. However, flipping one bit potentially may also change the value of the multiplexer map $\Gamma$. So we need to further refine the set $X$.)

We define the following two events with respect to an $x \in X$ $($ with $\Gamma(x)=(i, j))$ :

- $E_{1}(x): \eta(x) \neq C_{i, j}(\ell)$ for any $\ell \in[\sqrt{n}]\left(\right.$ thus $\left.C_{i, j}\left(x^{*}\right)=0\right)$;

- $E_{2}(x): T_{i^{\prime}}\left(x^{*}\right)=0$ for all $i^{\prime} \neq i \in[N]$.
We use $X^{\prime}$ to denote the set of strings $x \in X$ such that both $E_{1}(x)$ and $E_{2}(x)$ hold. The following claim shows that $\left(x, x^{*}\right)$ for every $x \in X^{\prime}$ is a violating edge of $f$.

Claim 1. For each $x \in X^{\prime},\left(x, x^{*}\right)$ is a violating edge of $f$.

Proof. It suffices to show that $f\left(x^{*}\right)=0$. As $x$ satisfies a unique term $T_{i}$ ( $T_{i}$ cannot have $\eta(x)$ as a variable as $x_{\eta(x)}=0$ ), it follows from $E_{2}(x)$ that $x^{*}$ uniquely satisfies $T_{i}$. It follows from $E_{1}(x)$ that $x^{*}$ uniquely falsifies $C_{i, j}$. As a result, $f\left(x^{*}\right)=h_{i, j}\left(x^{*}\right)=0$.

Furthermore, the violating edges $\left(x, x^{*}\right)$ induced by strings $x \in$ $X^{\prime}$ are indeed disjoint. (This is because, given $x^{*}$, one can uniquely reconstruct $x$ by locating $h_{i, j}$ using $\Gamma\left(x^{*}\right)$ and flipping the $k$ th bit of $x^{*}$ if $h_{i, j}$ is an anti-dictator function over the $k$ th variable.) Therefore, it suffices to show that $X^{\prime}$ (as a random set) has size $\Omega\left(2^{n}\right)$ with probability $\Omega(1)$, over choices $(\boldsymbol{T}, \boldsymbol{C}) \sim \mathcal{E}$ and $\boldsymbol{H} \sim \mathcal{E}_{\text {no. }}$. The lemma then follows from the characterization in Lemma 2.1.

Finally we work on the size of $X^{\prime}$. Fix a string $x \in\{0,1\}^{n}$ in the middle layers. The next claim shows that, when $(T, C) \sim \mathcal{E}$ and $H \sim \mathcal{E}_{\text {no }}, \boldsymbol{X}^{\prime}$ contain $x$ with $\Omega(1)$ probability.

CLAim 2. For any $x \in\{0,1\}^{n}$ with $(n / 2)-\sqrt{n} \leq|x| \leq(n / 2)+\sqrt{n}$,

$$
\underset{(T, C) \sim \mathcal{E}, H \sim \mathcal{E}_{n o}}{\operatorname{Pr}}\left[x \in X^{\prime}\right]=\Omega(1) .
$$

Proof. Fix an $x \in\{0,1\}^{n}$ in the middle layers. We calculate the probability of $x \in X^{\prime}$.

We partition the event of $x \in X^{\prime}$ into $\Theta\left(n N^{2}\right)$ subevents indexed by $i, j \in[N]$ and $k \in[n]$ with $x_{k}=0$. Each subevent corresponds to 1 ) Condition on $T$ : both $x$ and $x^{(k)}$ satisfy uniquely the $i$ th term; 2) Condition on $C$ : both $x$ and $x^{(k)}$ falsify uniquely the $j$ th term; 3 ) Condition on $\boldsymbol{H}: h_{i, j}$ is the anti-dictatorship function over the $k$ th variable. The probability of 3 ) is clearly $1 / n$.

The probability of 1) is at least

$$
\left(1-\left(\frac{n / 2+\sqrt{n}+1}{n}\right)^{\sqrt{n}}\right)^{N-1} \times\left(\frac{n / 2-\sqrt{n}}{n}\right)^{\sqrt{n}}=\Omega\left(\frac{1}{N}\right) \text {. }
$$

The probability of 2) is at least

$$
\left(1-\left(\frac{n / 2+\sqrt{n}}{n}\right)^{\sqrt{n}}\right)^{N-1} \times\left(\frac{n / 2-\sqrt{n}+1}{n}\right)^{\sqrt{n}}=\Omega\left(\frac{1}{N}\right) .
$$

Thus, the probability of $x \in X^{\prime}$ is $\Omega\left(n N^{2}\right) \times \Omega(1 / N) \times \Omega(1 / N) \times$ $\Omega(1 / n)=\Omega(1)$.

From Claim 2 and the fact that there are $\Omega\left(2^{n}\right)$ strings in the middle layer, the expected size of $\boldsymbol{X}^{\prime}$ is $\Omega\left(2^{n}\right)$. By Markov $\left|\boldsymbol{X}^{\prime}\right|=\Omega\left(2^{n}\right)$ with probability $\Omega(1)$. This finishes the proof.

Given Lemma 3.1 and 3.2, Theorem 1.1 follows directly from the following lemma which we show in the rest of the section. For the rest of the proof we fix the number of queries $q=n^{1 / 3} / \log ^{2} n$.

LEMMA 3.3. Let B be any q-query, deterministic algorithm with oracle access to $f$. Then we have

$$
\underset{f \sim \mathcal{D}_{\text {yes }}}{\operatorname{Pr}}[\text { accepts } f] \leq \underset{f \sim \mathcal{D}_{n o}}{\operatorname{Pr}}[\text { accepts } f]+o(1) .
$$


As $f$ is truncated in both distributions, we may assume WLOG that $B$ queries strings in the middle layers only (i.e., strings $x$ with $|x|$ between $(n / 2)-\sqrt{n}$ and $(n / 2)+\sqrt{n})$.

\subsection{Signatures and the New Oracle}

Let $(T, C)$ be a pair from the support of $\mathcal{E}$ and $H$ be a tuple from

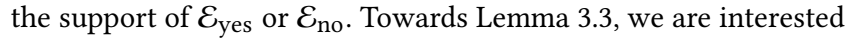
in deterministic algorithms that have oracle access to $f=f_{T, C, H}$ and attempt to distinguish $\mathcal{D}_{\text {yes }}$ from $\mathcal{D}_{\text {no }}$ (i.e., accept if $H$ is from $\mathcal{E}_{\text {yes }}$ and reject if it is from $\mathcal{E}_{\text {no }}$ ).

For convenience of our lower bound proof, we assume below that the oracle returns more than just $f(x)$ for each query $x \in$ $\{0,1\}^{n}$; instead of simply returning $f(x)$, the oracle returns a 4tuple $(\sigma, \tau, a, b)$ called the full signature of $x \in\{0,1\}^{n}$ with respect to $(T, C, H)$ (see Definition 3.5 below). It will become clear later that $f(x)$ can always be derived correctly from the full signature of $x$ and thus, query lower bounds against the new oracle carry over to the standard oracle. Once the new oracle is introduced, we may actually ignore the function $f$ and view any algorithm as one that has oracle access to the hidden triple $(T, C, H)$ and attempts to tell whether $H$ is from $\mathcal{E}_{\text {yes }}$ or $\mathcal{E}_{\text {no }}$.

We first give the syntactic definition of full signatures.

Definition 3.4. We let $\mathfrak{P}$ denote the set of all 4-tuples $(\sigma, \tau, a, b)$ with $\sigma \in\{0,1, *\}^{N}, \tau \in\{0,1, *\}^{N} \cup\{\perp\}$, and $a, b \in\{0,1, \perp\}$ satisfying the following properties:

(1) $\sigma$ is either 1) the all-0 string $0^{N}$;2) $e_{i}$ for some $i \in[N]$; or 3) $e_{i, i^{\prime}}$ for some $i<i^{\prime} \in[N]$.

(2) $\tau=\perp$ if $\sigma$ is of case 1) or 3). Otherwise (when $\sigma=e_{i}$ for some $i), \tau \in\{0,1, *\}^{N}$ is either 1) the all-1 string $1^{N}$;2) $\bar{e}_{j}$ for some $j \in[N]$; or 3$) \bar{e}_{j, j^{\prime}}$ for some $j<j^{\prime} \in[N]$.

(3) $a=b=\perp$ unless the following two cases happen: 1) If $\sigma=e_{i}$ and $\tau=\bar{e}_{j}$ for some $i, j \in[N]$, then $a \in\{0,1\}$ and $b=\perp$; or 2) If $\sigma=e_{i}$ and $\tau=\bar{e}_{j, j^{\prime}}$ for some $i \in[N]$ and $j<j^{\prime} \in[N]$, then $a, b \in\{0,1\}$.

We next define semantically the full signature of a string $x$ with respect to $(T, C, H)$.

Definition 3.5 (Full signature). We say $(\sigma, \tau, a, b)$ is the full signature of a string $x \in\{0,1\}^{n}$ with respect to $(T, C, H)$ if it satisfies the following properties:

(1) First, $\sigma \in\{0,1, *\}^{N}$ is determined by $T$ according to one of the following three cases: 1) $\sigma$ is the all-0 string $0^{N}$ if $T_{i}(x)=0$ for all $\left.i \in[N] ; 2\right)$ If there is a unique $i \in[N]$ with $T_{i}(x)=1$, then $\sigma=e_{i}$; or 3$)$ If there are more than one index $i \in[N]$ with $T_{i}(x)=1$, then $\sigma=e_{i, i^{\prime}}$ with $i<i^{\prime} \in[N]$ being the smallest two such indices. We call $\sigma$ the term signature of $x$.

(2) Second, $\tau=\perp$ if $\sigma$ is of case 1) or 3) above. Otherwise, assuming that $\sigma=e_{i}, \tau \in\{0,1, *\}^{N}$ is determined by $\left(C_{i, j}: j \in[N]\right)$, according to one of the following cases: 1) $\tau$ is the all-1 string $1^{N}$ if $C_{i, j}(x)=1$ for all $j \in[N]$; 2) If there is a unique $j$ with $C_{i, j}(x)=0$, then $\tau=\bar{e}_{j}$; or 3) If there are more than one index $j \in[N]$ with $C_{i, j}(x)=0$, then $\tau=\bar{e}_{j, j^{\prime}}$ with $j<j^{\prime} \in[N]$ being the smallest two such indices. We call $\tau$ the clause signature of $x$.
(3) Finally, $a=b=\perp$ unless: 1) If $\sigma=e_{i}$ and $\tau=\bar{e}_{j}$ for some $i, j$, then $a=h_{i, j}(x)$ and $b=\perp$; or 2) If $\sigma=e_{i}$ and $\tau=\bar{e}_{j, j^{\prime}}$ for some $i, j<j^{\prime} \in[N]$, then $a=h_{i, j}(x)$ and $b=h_{i, j^{\prime}}(x)$.

It follows from the definitions that the full signature of $x$ with respect to $(T, C, H)$ is in $\mathfrak{P}$. We also define the full signature of a set of strings $Q$ with respect to $(T, C, H)$.

Definition 3.6. The full signature (map) of a set $Q \subseteq\{0,1\}^{n}$ with respect to a triple $(T, C, H)$ is a map $\phi: Q \rightarrow \mathfrak{P}$ such that $\phi(x)$ is the full signature of $x$ with respect to $(T, C, H)$ for each $x \in Q$.

For simplicity, we will write $\phi(x)=\left(\sigma_{x}, \tau_{x}, a_{x}, b_{x}\right)$ to specify the term and clause signatures of $x$ as well as the values of $a$ and $b$ in the full signature $\phi(x)$ of $x$. Intuitively we may view $\phi$ as two levels of tables with entries in $\{0,1, *\}$. The (unique) top-level table "stacks" the term signatures $\sigma_{x}$, where each row corresponds to a string $x \in Q$ and each column corresponds to a term $T_{i}$ in $T$. In the second level a table appears for a term $T_{i}$ if the term signature of some string $x \in Q$ is $e_{i}$. In this case the second-level table at $T_{i}$ "stacks" the clause signatures $\tau_{x}$ for each $x \in Q$ with $\sigma_{x}=e_{i}$ where each row corresponds to such an $x$ and each column corresponds to a clause $C_{i, j}$ in $C$. (The number of columns is still $N$ since we only care about clauses $C_{i, j}, j \in[N]$, in the table at $T_{i}$.)

The lemma below shows that the new oracle is at least as powerful as the standard oracle. The proof can be found in [16].

Lemma 3.7. Let $(T, C)$ be from the support of $\mathcal{E}$ and $H$ from the support of $\mathcal{E}_{y e s}$ or $\mathcal{E}_{n o}$. Given any string $x \in\{0,1\}^{n}, f_{T, C, H}(x)$ is determined by its full signature with respect to $(T, C, H)$.

Given Lemma 3.7, it suffices to consider deterministic algorithms with the new oracle access to a hidden triple $(T, C, H)$, and Lemma 3.3 follows directly from the following lemma:

LEMMA 3.8. Let B be any q-query algorithm with the new oracle access to $(T, C, H)$. Then we have

$$
\begin{aligned}
\underset{(T, C) \sim \mathcal{E}, \boldsymbol{H} \sim \mathcal{E}_{y e s}}{\operatorname{Pr}}[\text { accepts }(T, C, H)] \\
\quad \leq \underset{(T, C) \sim \mathcal{E}, \boldsymbol{H} \sim \mathcal{E}_{n o}}{\operatorname{Pr}}[B \text { accepts }(T, C, H)]+o(1) .
\end{aligned}
$$

Such a deterministic algorithm $B$ can be equivalently viewed as a decision tree of depth $q$ (and we will abuse the notation to also denote this tree by $B$ ). Each leaf of the tree $B$ is labeled either "accept" or "reject." Each internal node $u$ of $B$ is labeled with a query string $x \in\{0,1\}^{n}$, and each of its outgoing edges $(u, v)$ is labeled a tuple from $\mathfrak{P}$. We refer to such a tree as a signature tree.

As the algorithm executes, it traverses a root-to-leaf path down the tree making queries to the oracle corresponding to queries in the nodes on the path. For instance at node $u$, after the algorithm queries $x$ and the oracle returns the full signature of $x$ with respect to the unknown $(T, C, H)$, the algorithm follows the outgoing edge $(u, v)$ with that label. Once a leaf $\ell$ is reached, $B$ accepts if $\ell$ is labelled "accept" and rejects otherwise.

Note that the number of children of each internal node is $|\mathfrak{P}|$, which is huge. Algorithms with the new oracle may adapt its queries to the full signatures returned by the oracle, while under the standard oracle, the queries may only adapt to the value of the function at previous queries. Thus, while algorithms making $q$ queries in the 
standard oracle model can be described by trees of size $2^{q}, q$-query algorithms with this new oracle have tree size $\left(2^{\Theta(\sqrt{n})}\right)^{q}$.

We associate each node $u$ in the tree $B$ with a map $\phi_{u}: Q_{u} \rightarrow \mathfrak{P}$ where $Q_{u}$ is the set of queries made along the path from the root to $u$ so far, and $\phi_{u}(x)$ is the label of the edge that the root-to- $u$ path takes after querying $x$. We will be interested in analyzing the following two probabilities: the probability of $(T, C, H)$ reaching $u$ when $(T, C) \sim \mathcal{E}$ and $\boldsymbol{H} \sim \mathcal{E}_{\text {yes }}$ versus the same probability when

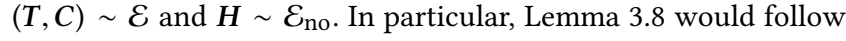
trivially if for every leaf $\ell$ of $B$ :

$$
\begin{aligned}
\underset{(T, C) \sim \mathcal{E}}{\operatorname{Pr}} \boldsymbol{H} \sim \mathcal{E}_{\text {yes }} & {[(T, C, H) \text { reaches } \ell] } \\
& \leq(1+o(1)) \cdot \underset{(T, C) \sim \mathcal{E}, H \sim \mathcal{E}_{\text {no }}}{\operatorname{Pr}}[(T, C, H) \text { reaches } \ell] .
\end{aligned}
$$

However, (4) above does not hold in general. Our plan for the rest of the proof is to prune an o(1)-fraction of leaves (measured in terms of their total probability under the yes-case) and show (4) for the rest. To better understand these probabilities, we need to first introduce some useful notation.

\subsection{Notation for Full Signature Maps}

Given a map $\phi: Q \rightarrow \mathfrak{P}$ for some set $Q \subseteq\{0,1\}^{n}$, we write $\phi(x)=$ $\left(\sigma_{x}, \tau_{x}, a_{x}, b_{x}\right)$ for each $x \in Q$ and use $\sigma_{x, i}, \tau_{x, j}$ to denote the $i$ th entry and $j$ th entry of $\sigma_{x}$ and $\tau_{x}$, respectively. Recall that $\tau_{x, j}$ is not defined if $\tau_{x}=\perp$. (Below we will only be interested in $\tau_{x, j}$ if $\sigma_{x}=e_{i}$ for some $i \in[N]$.)

We introduce the following notation for $\phi$. We say $\phi$ induces $a$ tuple $(I ; J ; P ; R ; A ; \rho)$, where

- $I \subseteq[N]$ is given by $I=\left\{i \in[N]: \exists x \in Q\right.$ with $\left.\sigma_{x, i}=1\right\}$. (So in terms of the first-level table, $I$ consists of columns that contain at least one 1-entry.)

- $J=\left(J_{i} \subseteq[N]: i \in I\right)$ is a tuple of sets indexed by $i \in I$. For each $i \in I$, we have

$J_{i}=\left\{j \in[N]: \exists x \in Q\right.$ with $\sigma_{x}=e_{i}$ and $\left.\tau_{x, j}=0\right\}$.

(In terms of the second-level table at $T_{i}, J_{i}$ consists of columns that contain at least one 0-entry.) By the definition of $\mathfrak{P}$, each $x$ with $\sigma_{x}=e_{i}$ can contribute at most two $j$ 's to $J_{i}$. Also $x$ does not contribute any $j$ to $J_{i}$ if $\sigma_{x}=e_{i, i^{\prime}}$ or $e_{i^{\prime}, i}$, in which case $\tau_{x}=\perp$, or if $\sigma_{x}=e_{i}$ but $\tau_{x}=1^{N}$. So in general $J_{i}$ can be empty for some $i \in I$.

- $P=\left(P_{i}, P_{i, j}: i \in I, j \in J_{i}\right)$ is a tuple of two types of subsets of $Q$, where $P_{i}=\left\{x \in Q: \sigma_{x, i}=1\right\}$ and

$$
P_{i, j}=\left\{x \in Q: \sigma_{x}=e_{i} \text { and } \tau_{x, j}=0\right\} .
$$

(In terms of the first-level table, $P_{i}$ consists of rows that are 1 on the $i$ th column; in terms of the second-level table at $T_{i}, P_{i, j}$ consists of rows that are 0 on the $j$ th column.) Both $P_{i}$ and $P_{i, j}$ are not empty by the definition of $I, J_{i}$.

- $R=\left(R_{i}, R_{i, j}: i \in I, j \in J_{i}\right)$ is a tuple of two types of subsets of $Q$, where $R_{i}=\left\{x \in Q: \sigma_{x, i}=0\right\}$ and

$$
R_{i, j}=\left\{x \in Q: \sigma_{x}=e_{i} \text { and } \tau_{x, j}=1\right\} .
$$

(In terms of the first-level table, $R_{i}$ consists of rows that are 0 on the $i$ th column; in terms of the second-level table at $T_{i}, R_{i, j}$ consists of rows that are 1 on the $j$ th column.)
- $A=\left(A_{i, 0}, A_{i, 1}, A_{i, j, 0}, A_{i, j, 1}: i \in I, j \in J_{i}\right)$ is a tuple of subsets of $[n]$. For $i \in I, j \in J_{i}$, we have

$$
\begin{aligned}
A_{i, 1} & =\left\{k \in[n]: \forall x \in P_{i}, x_{k}=1\right\} \text { and } \\
A_{i, j, 1} & =\left\{k \in[n]: \forall x \in P_{i, j}, x_{k}=1\right\}
\end{aligned}
$$

with $A_{i, 0}$ and $A_{i, j, 0}$ defined similarly using $x_{k}=0$ instead. All the sets are well-defined as $P_{i}$ and $P_{i, j}$ are not empty.

- $\rho=\left(\rho_{i, j}: i \in I, j \in J_{i}\right)$ is a tuple of Boolean functions $\rho_{i, j}: P_{i, j} \rightarrow\{0,1\}$. For each string $x \in P_{i, j}$, we have $\rho_{i, j}(x)=a_{x}$ if $\tau_{x}=\bar{e}_{j}$ or $\tau_{x}=\bar{e}_{j, j^{\prime}}$ for some $j^{\prime}>j$; $\rho_{i, j}(x)=b_{x}$ if $\tau_{x}=\bar{e}_{j^{\prime}, j}$ for some $j^{\prime}<j$.

Intuitively $I$ is the set of indices of terms with some string $x \in Q$ satisfying the term $T_{i}$ as reported in $\sigma_{x}$, and $P_{i}$ is the set of such strings while $R_{i}$ is the set of strings which do not satisfy $T_{i}$. For each $i \in I, J_{i}$ is the set of indices of clauses with some string $x \in P_{i}$ satisfying $T_{i}$ uniquely and falsifying the clause $C_{i, j} . P_{i, j}$ is the set of such strings, and $R_{i, j}$ is the set of strings which satisfy $T_{i}$ uniquely but also satisfy $C_{i, j}$. We collect the following facts which are immediate from the definition.

FACT 1 . Let $(I ; J ; P ; R ; A ; \rho)$ be the tuple induced by a map $\phi: Q \rightarrow$ $\Sigma$. Then we have

- $|I| \leq \sum_{i \in I}\left|P_{i}\right| \leq 2|Q|$.

- For each $i \in I,\left|J_{i}\right| \leq \sum_{j \in J_{i}}\left|P_{i, j}\right| \leq 2\left|P_{i}\right|$.

- For each $i \in I$ and $j \in J_{i},\left|R_{i}\right|$ and $\left|R_{i, j}\right|$ are at most $|Q|$.

- For each $i \in I$ and $j \in J_{i}$, we have $P_{i, j} \subseteq P_{i}, A_{i, 0} \subseteq A_{i, j, 0}$, and $A_{i, 1} \subseteq A_{i, j, 1}$.

Note that $|I|$ and $\sum_{i \in I}\left|J_{i}\right|$ can be strictly larger than $|Q|$, as some $x$ may satisfy more than one (but at most two) term with $\sigma_{x}=e_{i, i^{\prime}}$ and some $x$ may falsify more than one clause with $\tau_{x}=\bar{e}_{j, j^{\prime}}$.

The sets in $A$ are important for the following reasons that we summarize below.

FACT 2. Let $\phi: Q \rightarrow \mathfrak{P}$ be the full signature map of $Q$ with respect to $(T, C, H)$. Then

- For each $i \in I$, we have $T_{i}(k) \in A_{i, 1}$ for all $k \in[\sqrt{n}]$ and $T_{i}(x)=0$ for each $x \in R_{i}$.

- For each $i \in I$ and $j \in J_{i}$, we have $C_{i, j}(k) \in A_{i, j, 0}$ for all $k \in[\sqrt{n}]$ and $C_{i, j}(x)=1$ for each $x \in R_{i, j}$.

Before moving back to the proof, we introduce the following consistency condition on $P$.

Definition 3.9. Let $(I ; J ; P ; R ; A ; \rho)$ be the tuple induced by a map $\phi: Q \rightarrow \mathfrak{P}$. We say that $P_{i, j}$ for some $i \in I$ and $j \in J_{i}$ is 1 -consistent if $\rho_{i, j}(x)=1$ for all $x \in P_{i, j}$, and 0 -consistent if $\rho_{i, j}(x)=0$ for all $x \in P_{i, j}$; otherwise we say $P_{i, j}$ is inconsistent.

Let $\phi$ be the full signature map of $Q$ with respect to $(T, C, H)$. If $P_{i, j}$ is 1-consistent, the index $k$ of the variable $x_{k}$ in the dictatorship or anti-dictatorship function $h_{i, j}$ must lie in $A_{i, j, 0}$ (when $h_{i, j}$ is an anti-dictator) or $A_{i, j, 1}$ (when $h_{i, j}$ is a dictator); the situation is similar if $P_{i, j}$ is 0 -consistent but would be more complicated if $P_{i, j}$ is inconsistent. Below we prune an edge whenever some $P_{i, j}$ in $P$ becomes inconsistent. This way we make sure that $P_{i, j}$ 's in every leaf left are consistent. 


\subsection{Tree Pruning}

Consider an edge $(u, v)$ in $B$. Let $\phi_{u}: Q \rightarrow \mathfrak{P}$ and $\phi_{v}: Q \cup\{x\} \rightarrow \mathfrak{P}$ be the maps associated with $u$ and $v$, with $x$ being the query made at $u$ and $\phi_{v}(x)$ being the label of $(u, v)$. Let $(I ; J ; P ; R ; A ; \rho)$ and $\left(I^{\prime} ; J^{\prime} ; P^{\prime} ; R^{\prime} ; A^{\prime} ; \rho^{\prime}\right)$ be the two tuples induced by $\phi_{u}$ and $\phi_{v}$.

We list some easy facts about how $(I ; J ; P ; R ; A ; \rho)$ is updated to obtain $\left(I^{\prime} ; J^{\prime} ; P^{\prime} ; R^{\prime} ; A^{\prime} ; \rho^{\prime}\right)$.

FACT 3. Let $\phi_{v}(x)=\left(\sigma_{x}, \tau_{x}, a_{x}, b_{x}\right)$ for the $x$ queried at $u$. Then

- The string $x$ queried is placed in $P_{i}^{\prime}$ if $\sigma_{x, i}=1$, and is placed in $P_{i, j}^{\prime}$ if $\sigma_{x}=e_{i}$ and $\tau_{x, j}=0$.

- Each new set in $P^{\prime}$ (i.e., $P_{i}^{\prime}$ with $i \notin I$ or $P_{i, j}^{\prime}$ with either $i \notin I$ or $i \in I$ but $\left.j \notin J_{i}\right)$, if any, is $\{x\}$ and the corresponding $A_{i, 1}^{\prime}$ or $A_{i, j, 1}^{\prime}$ is $\left\{k: x_{k}=1\right\}$ and $A_{i, 0}^{\prime}$ or $A_{i, j, 0}^{\prime}$ is $\left\{k: x_{k}=0\right\}$.

- Each old set in $P^{\prime}$ (i.e., $P_{i}^{\prime}$ with $i \in I$ or $P_{i, j}^{\prime}$ with $i \in I$ and $j \in J_{i}$ ) either stays the same or has $x$ being added to the set. For the latter case, $\left\{k: x_{k}=0\right\}$ is removed from $A_{i, 1}$ or $A_{i, j, 1}$ and $\left\{k: x_{k}=1\right\}$ is removed from $A_{i, 0}$ or $A_{i, j, 0}$ to obtain the new sets in $A^{\prime}$.

Now we are ready to define a set of so-called bad edges of $B$, which will be used to prune $B$. In the rest of the proof we use $\alpha$ to denote a large enough positive constant.

Definition 3.10. An edge $(u, v)$ is called a bad edge if at least one of the following events occur at $(u, v)$ and none of these events occur along the path from the root to $u$ (letting $\phi_{u}$ and $\phi_{v}$ be the maps associated with $u$ and $v, x$ be the new query string at $u$, $(I ; J ; P ; R ; A ; \rho)$ and $\left(I^{\prime} ; J^{\prime} ; P^{\prime} ; R^{\prime} ; A^{\prime} ; \rho^{\prime}\right)$ be the tuples that $\phi_{u}$ and $\phi_{v}$ induce, respectively):

- For some $i \in I,\left|A_{i, 1} \backslash A_{i, 1}^{\prime}\right| \geq \alpha \sqrt{n} \log n$.

- For some $i \in I$ and $j \in J_{i},\left|A_{i, j, 0} \backslash A_{i, j, 0}^{\prime}\right| \geq \alpha \sqrt{n} \log n$.

- For some $i \in I$ and $j \in J_{i}, P_{i, j}$ is 0 -consistent but $P_{i, j}^{\prime}$ is inconsistent (meaning that $x$ is added to $P_{i, j}$ with $\rho_{i, j}(y)=0$ for all $y \in P_{i, j}$ but $\rho_{i, j}^{\prime}(x)=1$, instead of 0 ).

- For some $i \in I$ and $j \in J_{i}, P_{i, j}$ is 1 -consistent but $P_{i, j}^{\prime}$ is inconsistent (meaning that $x$ is added to $P_{i, j}$ with $\rho_{i, j}(y)=1$ for all $y \in P_{i, j}$ but $\rho_{i, j}^{\prime}(x)=0$, instead of 1$)$.

Moreover, a leaf $\ell$ is $b a d$ if one of the edges along the root-to- $\ell$ path is bad; $\ell$ is good otherwise.

The following key pruning lemma states that the probability of $(T, C, H)$ reaching a bad leaf of $B$ is $o(1)$, when $(T, C) \sim \mathcal{E}$ and $\boldsymbol{H} \sim \mathcal{E}_{\text {yes. }}$. We delay the proof to Section 3.6.

Lemma 3.11 (Pruning Lemma).

$$
\underset{(T, C) \sim \mathcal{E}, H \sim \mathcal{E}_{\text {yes }}}{\operatorname{Pr}}[(T, C, H) \text { reaches a bad leaf of } B]=o(1) .
$$

The pruning lemma allow us to focus on the good leaves $\ell$ of $B$ only. In particular we know that along the root-to- $\ell$ path the sets $A_{i, 1}$ and $A_{i, j, 0}$ each cannot shrink by more than $\alpha \sqrt{n} \log n$ with a single query (otherwise the path contains a bad edge and $\ell$ is a bad leaf which we ignore). Moreover every set $P_{i, j}$ in $P$ at the end must remain consistent (either 0 -consistent or 1 -consistent).

We use these properties to prove the following lemma in Section 3.5 for good leaves of $B$.
Lemma 3.12 (Good Leaves ARE Nice). For each good leaf $\ell$ of $B$,

$$
\begin{aligned}
\underset{(T, C) \sim \mathcal{E}, \boldsymbol{H} \sim \mathcal{E}_{\text {yes }}}{\operatorname{Pr}}[(\boldsymbol{T}, \boldsymbol{C}, \boldsymbol{H}) \text { reaches } \ell] \\
\leq(1+o(1)) \cdot \underset{(T, C) \sim \mathcal{E}, \boldsymbol{H} \sim \mathcal{E}_{n o}}{\operatorname{Pr}}[(\boldsymbol{T}, \boldsymbol{C}, \boldsymbol{H}) \text { reaches } \ell] .
\end{aligned}
$$

We can now combine Lemmas 3.11 and 3.12 to prove Lemma 3.8.

Proof of Lemma 3.8. Let $L$ be the leaves labeled "accept," and $L^{*} \subset L$ be the good leaves labeled "accept." We ignore $(T, C) \sim \mathcal{E}$ in the subscript below since it appears in every probability.

$$
\begin{aligned}
& \underset{H \sim \mathcal{E}_{\text {yes }}}{\operatorname{Pr}}[B \text { accepts }(\boldsymbol{T}, C, \boldsymbol{H})] \\
& =\sum_{\ell \in L} \operatorname{Pr}_{H \sim \mathcal{E}_{\text {yes }}}[(T, C, H) \text { reaches } \ell] \\
& \leq \sum_{\ell \in L^{*}} \operatorname{Pr}_{\boldsymbol{H} \sim \mathcal{E}_{\mathrm{yes}}}[(\boldsymbol{T}, \boldsymbol{C}, \boldsymbol{H}) \text { reaches } \ell]+o(1) \\
& \leq(1+o(1)) \cdot \sum_{\ell \in L^{*}} \operatorname{Pr}_{\boldsymbol{H} \sim \mathcal{E}_{\mathrm{no}}}[(\boldsymbol{T}, \boldsymbol{C}, \boldsymbol{H}) \text { reaches } \ell]+o(1) \\
& \leq \underset{H \sim \mathcal{E}_{\text {no }}}{\operatorname{Pr}}[B \text { accepts }(T, C, H)]+o(1),
\end{aligned}
$$

where (6) used Lemma 3.11 and (7) used Lemma 3.12.

\subsection{Proof of Lemma 3.12 for Good Leaves}

We prove Lemma 3.12 in this section. Let $\ell$ be a good leaf associated with $\phi_{\ell}$ and $(I ; J ; P ; R ; A ; \rho)$ be the tuple that $\phi_{\ell}$ induces. Note that along the root-to- $\ell$ path, when a set $A_{i, 0}, A_{i, 1}, A_{i, j, 0}, A_{i, j, 1}$ is created for the first time in $A$, its size is between $(n / 2) \pm \sqrt{n}$ (since all queries made by $B$ lie in the middle layers). As a result, it follows from Definition 3.10 that for $i \in I$ and $j \in J_{i}$ :

i) $\left|A_{i, 1}\right| \geq(n / 2)-O\left(\left|P_{i}\right| \cdot \sqrt{n} \log n\right)$ and $\left|A_{i, j, 0}\right| \geq(n / 2)-O\left(\left|P_{i, j}\right| \cdot \sqrt{n} \log n\right)$

ii) $\left|A_{i, 0}\right|,\left|A_{i, 1}\right|,\left|A_{i, j, 0}\right|,\left|A_{i, j, 1}\right| \leq(n / 2)+\sqrt{n}$;

iii) $P_{i, j}$ is consistent (either 1 -consistent or 0 -consistent).

Additionally, notice that $A_{i, 1} \subseteq A_{i, j, 1}$; thus from i) we have

$$
\left|A_{i, j, 1}\right| \geq\left|A_{i, 1}\right| \geq(n / 2)-O\left(\left|P_{i}\right| \cdot \sqrt{n} \log n\right) .
$$

We use these properties to prove the following two claims. The proofs are elementary and can be found in the full version [16].

Claim 3. For each $i \in I$ and $j \in J_{i}$, we have

$$
\left|A_{i, j, 1}\right| \geq(n / 2)-O\left(\left|P_{i, j}\right|^{2} \cdot \sqrt{n} \log n\right) .
$$

Claim 4. For each $i \in I$ and $j \in J_{i}$, we have

$$
|| A_{i, j, 1}|-| A_{i, j, 0}|| \leq O\left(\sqrt{n} \log n \cdot \min \left\{\left|P_{i, j}\right|^{2},\left|P_{i}\right|\right\}\right)
$$

We are now ready to prove Lemma 3.12 .

Proof of Lemma 3.12. Let $\ell$ be a good leaf and let $\phi: Q \rightarrow \mathfrak{P}$ be the map associated with $\ell$. Let $|\mathcal{E}|$ denote the support size of $\mathcal{E}$. We may rewrite the two probabilities in (5) as follows:

$$
\begin{aligned}
& \frac{1}{|\mathcal{E}|} \sum_{(T, C)} \operatorname{Pr}_{\boldsymbol{H} \sim \mathcal{E}_{\text {yes }}}[(T, C, \boldsymbol{H}) \text { reaches } \ell] \text { and } \\
& \frac{1}{|\mathcal{E}|} \sum_{(T, C)} \operatorname{Pr}_{\boldsymbol{H} \sim \mathcal{E}_{\text {no }}}[(T, C, \boldsymbol{H}) \text { reaches } \ell]
\end{aligned}
$$


where the sum is over the support of $\mathcal{E}$. Hence, it suffices to show that for each $(T, C)$ such that

$$
\underset{H \sim \mathcal{E}_{\text {yes }}}{\operatorname{Pr}}[(T, C, \boldsymbol{H}) \text { reaches } \ell]>0,
$$

we have the following inequality:

$$
\frac{\operatorname{Pr}_{\boldsymbol{H} \sim \mathcal{E}_{\text {no }}}[(T, C, \boldsymbol{H}) \text { reaches } \ell]}{\operatorname{Pr}_{\boldsymbol{H} \sim \mathcal{E}_{\text {yes }}}[(T, C, \boldsymbol{H}) \text { reaches } \ell]} \geq 1-o(1) .
$$

Fix a pair $(T, C)$ such that (9) holds. Recall that $(T, C, H)$ reaches $\ell$ if and only if the signature of each $x \in Q$ with respect to $(T, C, H)$ matches exactly $\phi(x)=\left(\sigma_{x}, \tau_{x}, a_{x}, b_{x}\right)$. Given (9), the term and clause signatures of $x$ are already known to match $\sigma_{x}$ and $\tau_{x}$ (otherwise the LHS of (9) is 0 ). The rest, i.e., $a_{x}$ and $b_{x}$ for each $x \in Q$, depends on $H=\left(h_{i, j}\right)$ only.

As $\ell$ is consistent, there is a $\rho_{i, j} \in\{0,1\}$ for each $P_{i, j}$ such that every $x \in P_{i, j}$ satisfies $h_{i, j}(x)=\rho_{i, j}$. These are the only conditions for $H$ to match $a_{x}$ and $b_{x}$ for each $x \in Q$, and as a result, below we give the conditions on $H=\left(h_{i, j}\right)$ for the triple $(T, C, H)$ to reach $\ell$ :

- For $\mathcal{E}_{\text {yes }},(T, C, H)$ reaches $\ell$, where $H=\left(h_{i, j}\right)$ and $h_{i, j}(x)=x_{k_{i, j}}$, if and only if $k_{i, j} \in A_{i, j, \rho_{i, j}}$ for each $i \in I$ and $j \in J_{i}$ (so that each $x \in P_{i, j}$ has $h_{i, j}(x)=\rho_{i, j}$ ).

- For $\mathcal{E}_{\text {no }},(T, C, H)$ reaches $\ell$, where $H=\left(h_{i, j}\right)$ and $h_{i, j}(x)=\overline{x_{k_{i, j}}}$, if and only if $k_{i, j} \in A_{i, j, 1-\rho_{i, j}}$ for each $i \in I$ and $j \in J_{i}$ (so that each $x \in P_{i, j}$ has $h_{i, j}(x)=\rho_{i, j}$ ).

With this characterization, we can rewrite the LHS of (10) as

$$
\prod_{i \in I, j \in J_{i}}\left(\frac{\left|A_{i, j, 1-\rho_{i, j}}\right|}{\left|A_{i, j, \rho_{i, j}}\right|}\right)=\prod_{i \in I, j \in J_{i}}\left(1+\frac{\left|A_{i, j, 1-\rho_{i, j}}\right|-\left|A_{i, j, \rho_{i, j}}\right|}{\left|A_{i, j, \rho_{i, j}}\right|}\right) \text {. }
$$

Thus, applying Claim 4 and noting that $\left|A_{i, j, \rho_{i, j}}\right| \leq n$ (whether $\rho_{i, j}=0$ or 1$)$, the LHS of $(10)$ is at least

$$
\begin{aligned}
\prod_{i \in I, j \in J_{i}}(1- & \left.O\left(\frac{\log n \cdot \min \left\{\left|P_{i, j}\right|^{2},\left|P_{i}\right|\right\}}{\sqrt{n}}\right)\right) \\
& \geq 1-O\left(\frac{\log n}{\sqrt{n}}\right) \sum_{i \in I, j \in J_{i}} \min \left\{\left|P_{i, j}\right|^{2},\left|P_{i}\right|\right\} .
\end{aligned}
$$

Since $\sum_{j}\left|P_{i, j}\right| \leq 2\left|P_{i}\right|, \sum_{j \in J_{i}} \min \left\{\left|P_{i, j}\right|^{2},\left|P_{i}\right|\right\}$ is maximized if $\left|J_{i}\right|=$ $2 \sqrt{\left|P_{i}\right|}$ and $\left|P_{i, j}\right|=\sqrt{\left|P_{i}\right|}$. As a result, we have

$$
\sum_{i \in I, j \in J_{i}} \min \left\{\left|P_{i, j}\right|^{2},\left|P_{i}\right|\right\} \leq \sum_{i \in I} 2\left|P_{i}\right|^{3 / 2} \leq O\left(q^{3 / 2}\right),
$$

because $\sum_{i}\left|P_{i}\right| \leq 2 q$. This finishes the proof of the lemma since $q$ is chosen to be $n^{1 / 3} / \log ^{2} n$.

\subsection{Proof of the Pruning Lemma}

Let $E$ be the set of bad edges as defined in Definition 3.10 (recall that if $(u, v)$ is a bad edge, then the root-to- $u$ path cannot have any bad edge). We split the proof of Lemma 3.11 into four lemmas, one lemma for each type of bad edges. To this end, we define four sets $E_{1}, E_{2}, E_{3}$ and $E_{4}$ (we follow the same notation of Definition 3.10): An edge $(u, v) \in E$ belongs to

(1) $E_{1}$ if $\left|A_{i, 1} \backslash A_{i, 1}^{\prime}\right| \geq \alpha \sqrt{n} \log n$ for some $i \in I$;

(2) $E_{2}$ if $\left|A_{i, j, 0} \backslash A_{i, j, 0}^{\prime}\right| \geq \alpha \sqrt{n} \log n$ for some $i \in I$ and $j \in J_{i}$;
(3) $E_{3}$ if it is not in $E_{2}$ and for some $i \in I$ and $j \in J_{i}, P_{i, j}$ is 0 -consistent but $P_{i, j}^{\prime}$ is inconsistent (when $(u, v) \in E_{3}$ and the above occurs, we say $(u, v)$ is $E_{3}$-bad at $\left.(i, j)\right)$;

(4) $E_{4}$ if it is not in $E_{1}$ or $E_{2}$ and for some $i \in I$ and $j \in J_{i}, P_{i, j}$ is 1 -consistent but $P_{i, j}^{\prime}$ is inconsistent (when $(u, v) \in E_{4}$ and the above occurs, we say $(u, v)$ is $E_{4}$-bad at $\left.(i, j)\right)$.

We have $E=E_{1} \cup E_{2} \cup E_{3} \cup E_{4}$. (These four sets are not necessarily pairwise disjoint though we did exclude edges of $E_{2}$ from $E_{3}$ and edges of $E_{1}$ and $E_{2}$ from $E_{4}$ explicitly.) Each lemma below states that the probability of $(T, C) \sim \mathcal{E}$ and $\boldsymbol{H} \sim \mathcal{E}_{\text {yes }}$ taking an edge in $E_{i}$ is $o(1)$. Lemma 3.11 then follows directly from a union bound over the four sets.

Lemma 3.13. The probability of $(T, C) \sim \mathcal{E}$ and $\boldsymbol{H} \sim \mathcal{E}_{\text {yes }}$ taking an edge in $E_{1}$ is o(1).

Proof. Let $u$ be an internal node. We prove that, when $(T, C) \sim$ $\mathcal{E}$ and $\boldsymbol{H} \sim \mathcal{E}_{\text {yes }}$, either $(\boldsymbol{T}, \boldsymbol{C}, \boldsymbol{H})$ reaches $u$ with probability 0 or

$\underset{\mathcal{P r}}{\operatorname{Pr}} \sim \mathcal{E}_{\text {yes }}\left[(T, C, H)\right.$ takes an $E_{1}$-edge at $u \mid(T, C, H)$ reaches $\left.u\right]$ is $o(1 / q)$. Lemma 3.13 follows from Lemma 2.2 .

Below we assume that the probability of $(T, C, H)$ reaching node $u$ is positive. Let $\phi: Q \rightarrow \mathfrak{P}$ be the map associated with $u$, and let $x \in\{0,1\}^{n}$ be the string queried at $u$. Whenever we discuss a child node $v$ of $u$ below, we use $\phi^{\prime}: Q \cup\{x\} \rightarrow \mathfrak{P}$ to denote the map associated with $v$ and $(I ; J ; P ; R ; A ; \rho)$ and $\left(I^{\prime} ; J^{\prime} ; P^{\prime} ; R^{\prime} ; A^{\prime} ; \rho^{\prime}\right)$ to denote the tuples $\phi$ and $\phi^{\prime}$ induce. (Note that $v$ is not a specific node but can be any child of $u$.)

Fix an $i \in I$. We upperbound by $o\left(1 / q^{2}\right)$ the conditional probability of $(T, C, H)$ following a $(u, v)$ with $\left|A_{i, 1} \backslash A_{i, 1}^{\prime}\right| \geq \alpha \sqrt{n} \log n$. It follows from a union bound over $i \in I$ that the probability we are interested in is $o(1 / q)$.

With $i$ fixed, notice that any edge $(u, v)$ has either $A_{i, 1}^{\prime}=A_{i, 1}$ or $A_{i, 1}^{\prime}=A_{i, 1} \backslash \Delta_{i}$ with $\Delta_{i}=\left\{\ell \in A_{i, 1}: x_{\ell}=0\right\} \subseteq A_{i, 1}$, and the latter occurs if and only if $P_{i}^{\prime}=P_{i} \cup\{x\}$. Therefore, we assume WLOG that $\left|\Delta_{i}\right| \geq \alpha \sqrt{n} \log n$ (otherwise the conditional probability is 0 for $i$ ), and it suffices to bound by $o\left(1 / q^{2}\right)$ the conditional probability of $(\boldsymbol{T}, \boldsymbol{C}, \boldsymbol{H})$ taking an edge $(u, v)$ with $P_{i}^{\prime}=P_{i} \cup\{x\}$.

To analyze this conditional probability for $i \in I$, we fix a triple $\left(T_{-i}, C, H\right)$, where we use $T_{-i}$ to denote a sequence of $N-1$ terms with only the $i$ th term missing, such that

$$
\operatorname{Pr}_{T_{i}}\left[\left(\left(T_{-i}, T_{i}\right), C, H\right) \text { reaches } u\right]>0,
$$

where $T_{i}$ is a term drawn uniformly at random. It suffices to prove for any such $\left(T_{-i}, C, H\right)$ :

$$
\begin{aligned}
\operatorname{Pr}_{i} & {\left[\left(\left(T_{-i}, T_{i}\right), C, H\right) \text { reaches } u \text { and } P_{i}^{\prime}=P_{i} \cup\{x\}\right] } \\
\leq o\left(1 / q^{2}\right) \cdot \operatorname{Tr}_{i} & \left.\operatorname{Pr}\left[\left(T_{-i}, T_{i}\right), C, H\right) \text { reaches } u\right] .
\end{aligned}
$$

Recalling Fact 2, the latter event, $\left(\left(T_{-i}, T_{i}\right), C, H\right)$ reaching $u$, imposes the following two conditions on $T_{i}$ :

(1) For each $y \in P_{i}, T_{i}(y)=1$, and

(2) For each $z \in R_{i}, T_{i}(z)=0$.

Let $U$ be the set of all such terms $T: \sqrt{n} \rightarrow[n]$. Equivalently $T \in U$ iff $T(k) \in A_{i, 1}$ for all $k \in[\sqrt{n}]$ and every $z \in R_{i}$ has $z_{T(k)}=0$ for some $k \in[\sqrt{n}]$. Now regarding the former event in (11), a necessary 
condition over $T_{i}$ is the same as above but in addition we require $T_{i}(x)=1$. (Note that this is not a sufficient condition since for that we also need $T_{i}$ to be one of the first two terms that $x$ satisfies, which depends on $T_{-i}$.) Let $V$ denote the set of all such terms. Then $T \in V$ iff $T(k) \in A_{i, 1} \backslash \Delta_{i}$ for all $k \in[\sqrt{n}]$ and every $z \in R_{i}$ has $z_{T(k)}=0$ for some $k \in[\sqrt{n}]$.

In the rest of the proof we prove that $|V| /|U|=o\left(1 / q^{2}\right)$, from which (11) follows. Let $\ell=\log n$. First we write $U^{\prime}$ to denote the following subset of $U: T^{\prime} \in U$ is in $U^{\prime}$ if

$$
\left|\left\{k \in[\sqrt{n}]: T^{\prime}(k) \in \Delta_{i}\right\}\right|=\ell,
$$

and it suffices to show that $|V| /\left|U^{\prime}\right|=o\left(1 / q^{2}\right)$. Next we define the following bipartite graph $G$ between $U^{\prime}$ and $V$ (inspired by similar arguments of [6]): $T^{\prime} \in U^{\prime}$ and $T \in V$ have an edge iff $T^{\prime}(k)=T(k)$ for all $k \in[\sqrt{n}]$ with $T^{\prime}(k) \notin \Delta_{i}$. Each $T^{\prime} \in U^{\prime}$ has degree at most $\left|A_{i, 1} \backslash \Delta_{i}\right|^{\ell}$, as one can only move each $T^{\prime}(k) \in \Delta_{i}$ to $A_{i, 1} \backslash \Delta_{i}$.

To lowerbound the degree of a $T \in V$, note that one only needs at most $q$ many variables of $T$ to kill all strings in $R_{i}$. Let $H \subset[\sqrt{n}]$ be any set of size at most $q$ such that for each string $z \in R_{i}$, there exists a $k \in H$ with $z_{T(k)}=0 .{ }^{6}$ Then one can choose any $\ell$ distinct indices $k_{1}, \ldots, k_{\ell}$ from $\vec{H}$, as well as any $\ell$ (not necessarily distinct) variables $t_{1}, \ldots, t_{\ell}$ from $\Delta_{i}$, and let $T^{\prime}$ be a term where

$$
T^{\prime}(k)= \begin{cases}t_{i} & k=k_{i} \text { for some } i \in[\ell] \\ T(k) & \text { otherwise. }\end{cases}
$$

The resulting $T^{\prime}$ is in $U^{\prime}$ and $\left(T, T^{\prime}\right)$ is an edge in $G$. As a result, the degree of $T \in V$ is at least

$$
\left(\begin{array}{c}
\sqrt{n}-q \\
\ell
\end{array}\right) \cdot\left|\Delta_{i}\right|^{\ell}
$$

By counting the number of edges in $G$ in two different ways and using $\left|A_{i, 1}\right| \leq(n / 2)+\sqrt{n}$,

$\frac{\left|U^{\prime}\right|}{|V|} \geq\left(\begin{array}{c}\sqrt{n}-q \\ \ell\end{array}\right) \cdot\left(\frac{\left|\Delta_{i}\right|}{\left|A_{i, 1} \backslash \Delta_{i}\right|}\right)^{\ell} \geq\left(\frac{\sqrt{n}}{2 \ell} \cdot \frac{\alpha \sqrt{n} \ell}{(n / 2)+\sqrt{n}}\right)^{\ell}>\omega\left(q^{2}\right)$,

by choosing a large enough constant $\alpha>0$. This finishes the proof of the lemma.

Lemma 3.14. The probability of $(T, C) \sim \mathcal{E}$ and $\boldsymbol{H} \sim \mathcal{E}_{\text {yes }}$ taking an edge in $E_{2}$ is o(1).

Proof. The proof is similar to that of Lemma 3.13. Let $u$ be any internal node of the tree. We show that as $(T, C) \sim \mathcal{E}$ and $\boldsymbol{H} \sim \mathcal{E}_{\text {yes }}$, either $(\boldsymbol{T}, \boldsymbol{C}, \boldsymbol{H})$ reaches $u$ with probability 0 or

$$
\underset{(T, C) \sim \mathcal{E}, H \sim \mathcal{E}_{\text {yes }}}{\operatorname{Pr}}\left[(T, C, H) \text { takes an } E_{2} \text {-edge at } u \mid(T, C, H) \text { reaches } u\right]
$$

is $o(1 / q)$. Assume below WLOG that the probability of $(\boldsymbol{T}, \boldsymbol{C}, \boldsymbol{H})$ reaching $u$ is positive.

Fix $i \in I$ and $j \in J_{i}$. We upperbound the conditional probability of $(\boldsymbol{T}, \boldsymbol{C}, \boldsymbol{H})$ taking an edge $(u, v)$ with $\left|A_{i, j, 0} \backslash A_{i, j, 0}^{\prime}\right| \geq \alpha \sqrt{n} \log n$ by $o\left(1 / q^{3}\right)$. It follows from a union bound that the probability we are interested in is $o(1 / q)$. Similarly let

$$
\Delta_{i, j}=\left\{\ell \in A_{i, j, 0}: x_{\ell}=1\right\} \subseteq A_{i, j, 0},
$$

and assume WLOG that $\left|\Delta_{i, j}\right| \geq \alpha \sqrt{n} \log n$ (as otherwise the conditional probability is 0 for $i, j$ ). Then it suffices to upperbound the

\footnotetext{
${ }^{6}$ For example, since $\left|R_{i}\right| \leq q$, one can set $H$ to contain the smallest $k \in[\sqrt{n}]$ such that $z_{T(k)}=0$, for each $z \in R_{i}$.
}

conditional probability of $(\boldsymbol{T}, \boldsymbol{C}, \boldsymbol{H})$ going along an edge $(u, v)$ with $P_{i, j}^{\prime}=P_{i, j} \cup\{x\}$ by $o\left(1 / q^{3}\right)$. The rest of the proof is symmetric to that of Lemma 3.13 .

Lemma 3.15. The probability of $(T, C) \sim \mathcal{E}$ and $\boldsymbol{H} \sim \mathcal{E}_{\text {yes }}$ taking an edge in $E_{3}$ is o(1).

Proof. We fix any pair $(T, C)$ from the support of $\mathcal{E}$ and prove

$$
\underset{H \sim \mathcal{E}_{\text {yes }}}{\operatorname{Pr}}\left[(T, C, H) \text { takes an } E_{3} \text {-edge }\right]=o(1) .
$$

The lemma follows by averaging $(13)$ over all pairs $(T, C)$ in the support of $\mathcal{E}$. To prove (13) we fix any internal node $u$ such that the probability of $(T, C, H)$ reaching $u$ is positive, and prove that

$\underset{H \sim \mathcal{E}_{\text {yes }}}{\operatorname{Pr}}\left[(T, C, \boldsymbol{H})\right.$ takes an $E_{3}$-edge leaving $u \mid(T, C, \boldsymbol{H})$ reaches $\left.u\right]$

is $o(1 / q)$. (13) follows by Lemma 2.2. Below we assume the probability of $(T, C, \boldsymbol{H})$ reaching $u$ is positive.

We assume WLOG that there is no edge in $E$ along the root-to- $u$ path; otherwise, (14) is 0 .

We follow the same notation used in the proof of Lemma 3.13, i.e., $\phi_{u}: Q \rightarrow \mathfrak{P}$ as the map associated with $u, x$ as the query made at $u$, and $(I ; J ; P ; R ; A ; \rho)$ as the tuple induced by $\phi_{u}$. We also write $F$ to denote the set of pairs $(i, j)$ such that $i \in I$ and $j \in J$.

Observe that since $(T, C)$ is fixed, the term and clause signatures of every string are fixed, and in particular the term and clause signatures (denoted $\sigma_{x}$ and $\tau_{x}$ ) of $x$ are fixed. We assume WLOG that $\sigma_{x}=e_{k}$ for some $k \in[N]$ (otherwise $x$ will never be added to any $P_{i, j}$ when $(T, C, \boldsymbol{H})$ leaves $u$ and (14) is 0 by the definition of $\left.E_{3}\right)$. In this case we write $D$ to denote the set of $\left\{(k, j): \tau_{x, j}=0\right\}$ with $|D| \leq 2$. As a result, whenever $(T, C, \boldsymbol{H})$ takes an $E_{3}$-edge leaving from $u$, this edge must be $E_{3}$-bad at one of the pairs $(k, j) \in D$. Thus, the LHS of (14) is the same as

$$
\begin{array}{r}
\sum_{(k, j) \in D} \operatorname{Pr}_{\boldsymbol{H} \sim \mathcal{E}_{\text {yes }}}\left[(T, C, \boldsymbol{H}) \text { takes a }(u, v) \text { that is } E_{3}\right. \text {-bad } \\
\text { at }(k, j) \mid(T, C, \boldsymbol{H}) \text { reaches } u] .
\end{array}
$$

To bound the conditional probability for $(k, j)$ above by $o(1 / q)$, we assume WLOG that $(k, j) \in F$ (otherwise $x$ would create a new $P_{k, j}$ whenever $(T, C, \boldsymbol{H})$ takes an edge $(u, v)$ that leaves $u$, and the latter cannot be $E_{3}$-bad at $\left.(k, j)\right)$. Next we define $\left(A_{k, j, 0}\right.$ below is well defined since $(k, j) \in F)$

$$
\Delta_{k, j}=\left\{\ell \in A_{k, j, 0}: x_{\ell}=1\right\} \text {. }
$$

We may assume that $\left|\Delta_{k, j}\right|<\alpha \sqrt{n} \log n$; otherwise, $(T, C, H)$ can never take a $(u, v)$ in $E_{3}$ as $E_{2}$-edges are explicitly excluded from $E_{3}$. Finally, we assume WLOG $\rho_{k, j}(y)=0$ for all $y \in P_{k, j}$; otherwise, the edge $(u, v)$ that $(T, C, \boldsymbol{H})$ takes can never be $E_{3}$-bad at $(k, j)$.

With all these assumptions on $(k, j)$ in place, we prove the following inequality:

$$
\begin{aligned}
\underset{H \sim \mathcal{E}_{\text {yes }}}{\operatorname{Pr}}\left[(T, C, H) \text { takes a }(u, v) \text { that is } E_{3} \text {-bad at }(k, j)\right] \\
\leq \frac{\left|\Delta_{k, j}\right|}{\left|A_{k, j, 0}\right|} \cdot \underset{H \sim \mathcal{E}_{\text {yes }}}{\operatorname{Pr}}[(T, C, H) \text { reaches } u] .
\end{aligned}
$$

Given $\left|\Delta_{k, j}\right|=O(\sqrt{n} \log n)$ and $\left|A_{i, j, 0}\right| \geq(n / 2)-O(q \sqrt{n} \log n)=$ $\Omega(n)$ (since there is no bad edge particularly no $E_{2}$-edge, from the root to $u$ ), (14) follows by summing over $D$, with $|D| \leq 2$. 
We work on (16) in the rest of the proof. Fix any tuple $H_{-(k, j)}$ (with its $(k, j)$ th entry missing) such that the probability of $(T, C$, $\left.\left(H_{-(k, j)}, \boldsymbol{h}\right)\right)$ reaching $u$ is positive, where $\boldsymbol{h}$ is a random dictator function with its dictator variable drawn from $[n]$ uniformly. Then (16) follows from

$$
\begin{array}{r}
\underset{\boldsymbol{h}}{\operatorname{Pr}}\left[\left(T, C,\left(H_{-(k, j)}, \boldsymbol{h}\right)\right) \text { takes }(u, v) \text { that is } E_{3} \text {-bad at }(k, j)\right] \\
\leq \frac{\left|\Delta_{k, j}\right|}{\left|A_{k, j, 0}\right|} \cdot \underset{\boldsymbol{h}}{\operatorname{Pr}}\left[\left(T, C,\left(H_{-(k, j)}, \boldsymbol{h}\right)\right) \text { reaches } u\right] .
\end{array}
$$

The event on the RHS, i.e., $\left(T, C,\left(H_{-(k, j)}, \boldsymbol{h}\right)\right)$ reaching $u$, imposes the following condition on $r$, the dictator variable of $\boldsymbol{h}: r \in A_{k, j, 0}$, since $\rho_{k, j}(y)=0$ for all $y \in P_{k, j}$. Hence the probability on the RHS of (17) is $\left|A_{i, j, 0}\right| / n$. On the other hand, the event on the LHS of (17), that $\left(T, C,\left(H_{-(i, j)}, \boldsymbol{h}\right)\right)$ follows a $(u, v)$ that is $E_{3}$-bad at $(k, j)$, imposes the following necessary condition for $r: r \in \Delta_{k, j} \cdot{ }^{7}$ So the probability on the LHS of (17) is at most $\left|\Delta_{k, j}\right| / n$. (17) follows.

Lemma 3.16. The probability of $(T, C) \sim \mathcal{E}$ and $\boldsymbol{H} \sim \mathcal{E}_{\text {yes }}$ taking an edge in $E_{4}$ is o(1).

Proof. We fix a pair $(T, C)$ from the support of $\mathcal{E}$ and prove

$$
\underset{H \sim \mathcal{E}_{\text {yes }}}{\operatorname{Pr}}\left[(T, C, H) \text { takes an } E_{4} \text {-edge }\right]=o(1) \text {. }
$$

The lemma follows by averaging $(18)$ over all $(T, C)$ in the support of $\mathcal{E}$. To prove (18), fix a leaf $\ell$ such that the probability of $(T, C, \boldsymbol{H})$ reaching $\ell$ is positive. Let $u_{1} \cdots u_{t^{\prime}} u_{t^{\prime}+1}=\ell$ be the root-to- $\ell$ path and let $q\left(u_{s}\right)$ denote the following conditional probability:

$\underset{\boldsymbol{H} \sim \mathcal{E}_{\text {yes }}}{\operatorname{Pr}}\left[(T, C, \boldsymbol{H})\right.$ takes an $E_{4}$-edge leaving $u_{s} \mid(T, C, \boldsymbol{H})$ reaches $\left.u_{s}\right]$. It then suffices to show for every such leaf $\ell$,

$$
\sum_{s \in\left[t^{\prime}\right]} q\left(u_{s}\right)=o(1)
$$

since (18) would then follow by Lemma 2.3. To prove (19), we use $t$ to denote the smallest integer such that $\left(u_{t+1}, u_{t+2}\right)$ is an edge in $E_{1}$ or $E_{2}$ with $t=t^{\prime}$ by default if there is no such edge along the path. By the choice of $t$, there is no edge in $E_{1}$ or $E_{2}$ along $u_{1} \cdots u_{t+1}$. For (19) it suffices to show

$$
\sum_{s \in[t]} q\left(u_{s}\right)=o(1) .
$$

To see this we consider two cases. If there is no $E_{1}, E_{2}$ edge along the root-to- $\ell$ path, then the two sums in (19) and (20) are the same. If $\left(u_{t+1}, u_{t+2}\right)$ is an edge in $E_{1}$ or $E_{2}$, then $q\left(u_{s}\right)=0$ if $s \geq t+2$ (since $(u, v) \notin E$ if there is already an edge in $E$ along the path to $u$ ). We claim that $q\left(u_{t+1}\right)$ must be 0 as well. This is because, given that $(T, C)$ is fixed and that $(T, C, \boldsymbol{H})$ takes $\left(u_{t+1}, u_{t+2}\right)$ with a positive probability, whenever $(T, C, \boldsymbol{H})$ follows an edge $\left(u_{t+1}, v\right)$ from $u_{t+1}$, $v$ has the same term and clause signatures $\left(\sigma_{x}, \tau_{x}\right)$ as $u_{t+2}$ and thus, also has the same $P$ and $A$ (as part of the tuple its map induces). As a result $\left(u_{t+1}, v\right)$ is also in $E_{1}$ or $E_{2}$ and cannot be an edge in $E_{4}$ (recall that we explicitly excluded $E_{1}$ and $E_{2}$ from $E_{4}$ ). Below we focus on $u_{s}$ with $s \in[t]$ and upperbound $q\left(u_{s}\right)$.

For each $s \in[t]$ we use $x^{s}$ to denote the string queried at $u_{s}$ and let $\left(I^{s} ; J^{s} ; P^{s} ; Q^{s} ; R^{s} ; \rho^{s}\right)$ be the tuple induced by the map associated with $u_{s}$. We also write $F_{s}$ to denote the set of pairs $(i, j)$ with $i \in$

\footnotetext{
${ }^{7}$ This is not sufficient as the other pair $\left(k, j^{\prime}\right) \in D$ may have $\left|\Delta_{k, j^{\prime}}\right| \geq \alpha \sqrt{n} \log n$.
}

$I^{s}, j \in J_{i}^{s}$. Following the same arguments used to derive (15) in the proof of Lemma 3.15, let $D_{S} \subseteq F_{S}$ denote the set of at most two pairs $(i, j)$ such that $x^{s}$ is added to $P_{i, j}^{s}$ when $(T, C, \boldsymbol{H})$ reaches $u_{s}$. Note that if $x^{s}$ just creates a new $P_{i, j}$ (so $(i, j) \notin F_{s}$ ), we do not include it in $D_{s}$. As a result, whenever $(T, C, \boldsymbol{H})$ takes an $E_{4}$-edge $(u, v)$, the latter must be $E_{4}$-bad at one of $(i, j) \in D_{s}$.

Next for each $(i, j) \in D_{s}$, we follow (16) to show that

$$
\begin{array}{r}
\underset{\boldsymbol{H} \sim \mathcal{E}_{\text {yes }}}{\operatorname{Pr}}\left[(T, C, \boldsymbol{H}) \text { takes a }(u, v) \text { that is } E_{4} \text {-bad at }(i, j)\right] \\
\leq \frac{\left|\Delta_{i, j}^{s}\right|}{\left|A_{i, j, 1}^{s}\right|} \cdot \operatorname{Pr}_{\boldsymbol{H} \sim \mathcal{E}_{\text {yes }}}[(T, C, \boldsymbol{H}) \text { reaches } u],
\end{array}
$$

where $\Delta_{i, j}^{s}$ is defined as $\Delta_{i, j}^{s}=\left\{k \in A_{i, j, 1}^{s}: x_{k}^{s}=0\right\}$. As there is no $E_{1}$ or $E_{2}$ edge along the path to $u_{s},\left|A_{i, j, 1}^{s}\right|=\Omega(n)$ by (8). Thus,

$$
\begin{aligned}
q\left(u_{s}\right) & \leq O(1 / n) \cdot \sum_{(i, j) \in D_{s}}\left|\Delta_{i, j}^{s}\right| \quad \text { and } \\
\sum_{s \in[t]} q\left(u_{s}\right) & \leq O(1 / n) \cdot \sum_{s \in[t]} \sum_{(i, j) \in D_{s}}\left|\Delta_{i, j}^{s}\right| .
\end{aligned}
$$

Let $\left(I^{*} ; J^{*} ; P^{*} ; R^{*} ; A^{*} ; \rho^{*}\right)$ be the tuple induced by the map associated with $u_{t+1}$ and let $F^{*}$ be the set of $(i, j)$ with $i \in I^{*}$ and $j \in J_{i}^{*}$. We upperbound the second sum in (21) above by focusing on any fixed pair $(i, j) \in F^{*}$ and observing that

$$
\sum_{s:(i, j) \in D_{s}}\left|\Delta_{i, j}^{s}\right|+\left|A_{i, j, 1}^{*}\right| \leq(n / 2)+\sqrt{n}
$$

This is because $\Delta_{i, j}^{s}$ and $A_{i, j, 1}^{*}$ are pairwise disjoint and their union is indeed exactly the number of 1-entries of the query string along the path that first creates $P_{i, j}$. The latter is at most $(n / 2)+\sqrt{n}$ as we assumed that strings queried in the tree lie in the middle layers. On the other hand,

$$
\left|A_{i, j, 1}^{*}\right| \geq(n / 2)-O\left(\sqrt{n} \log n \cdot \min \left\{\left|P_{i, j}^{*}\right|^{2},\left|P_{i}^{*}\right|\right\}\right) .
$$

This follows directly from (8) and Claim 3 and our choice of $t$ at the beginning of the proof so that there is no $E_{1}$ or $E_{2}$ edge from $u_{1}$ to $u_{t+1}$. We finish the proof by plugging the two inequalities into (21) and follow the same arguments used at the end of the proof of the lemma for good leaves.

\section{TIGHTNESS OF DISTRIBUTIONS}

In this section we provide the reader with some intuition of why the analyses of [6] and this paper are tight. In particular, we sketch onesided algorithms to find violating pairs in the far-from-monotone functions from the distributions considered. We maintain this discussion at a high level.

\subsection{An Algorithm for Distributions of [6]}

Belovs and Blais define a pair of distributions $\mathcal{D}_{\text {yes }}^{*}$ and $\mathcal{D}_{\text {no }}^{*}$ over functions of $n$ variables. To define $\mathcal{D}_{\text {yes }}^{*}$ and $\mathcal{D}_{\text {no }}^{*}$, recall Talagrand's random DNF [32] (letting $N=2^{\sqrt{n}}$ ): a function $f$ drawn from Tal is the disjunction of $N$ terms $T_{i}, i \in[N]$, each $T_{i}$ being the conjunction of $\sqrt{n}$ variables sampled independently and uniformly from $[n]$.

Next we use Tal to define $\mathrm{Tal}_{ \pm}$. To draw a function $\boldsymbol{g}$ from $\mathrm{Tal}_{ \pm}$, one samples an $f$ from Tal and a random $\sqrt{n}$-subset $S$ of $[n] .{ }^{8}$ Then

${ }^{8}$ Formally, $S$ is sampled by including each element of $[n]$ independently with probability $1 / \sqrt{n}$. 
$\boldsymbol{g}(x)=f\left(x^{(S)}\right)$. Equivalently variables in $T_{i} \cap S$ appear negated in the conjunction of $T_{i}$. The $\mathcal{D}_{\text {yes }}^{*}$ distribution is then the truncation of Tal, and the $\mathcal{D}_{\text {no }}^{*}$ distribution is the truncation of $\mathrm{Tal}_{ \pm}$. Every $f \sim \mathcal{D}_{\text {yes }}^{*}$ is monotone by definition; [6] shows that $g \sim \mathcal{D}_{\text {no }}^{*}$ is far from monotone by using the extremal noise sensitivity property of Talagrand functions [27].

We now sketch a $O\left(n^{1 / 4}\right)$-query one-sided algorithm that rejects $\boldsymbol{g} \sim \mathcal{D}_{\text {no }}^{*}$ with high probability. Note that the description below is not a formal analysis; the goal is to discuss the main idea behind the algorithm. Let $g$ be a function in the support of $\mathcal{D}_{\text {no }}^{*}$ defined by $T_{i}$ and $S$ with $T_{i}^{\prime}=T_{i} \backslash S$. Then the algorithm starts by sampling a random $x \in\{0,1\}^{n}$ in the middle layers with $g(x)=1$. It is likely $(\Omega(1)$ probability by a simple calculation) that:

(1) $x$ satisfies a unique term $T_{k}^{\prime}$ among all $T_{i}^{\prime}$ s.

(2) $T_{k} \cap S$ contains a unique $\ell \in[n]$ (by 1 ).

(3) $T_{k}=T_{k}^{\prime} \cup\{\ell\}$ and $x$ has $x_{\ell}=0($ since $g(x)=1)$.

Assume this is the case, and let $A_{0}$ and $A_{1}$ denote the set of 0 -indices and 1-indices of $x$, respectively. Then $T_{k}^{\prime} \subseteq A_{1}$ and $\ell \in A_{0}$.

The first stage of the algorithm goes as follows:

Stage 1. Repeat the following for $n^{1 / 4}$ times: Pick a random subset $R \subset A_{1}$ of size $\sqrt{n}$ and query $g\left(x^{(R)}\right)$. By

(1) and (2), $g\left(x^{(R)}\right)=1$ iff $R \cap T_{k}^{\prime}=\emptyset$, which happens with $\Omega(1)$ probability. Let $A_{1}^{\prime}$ denote $A_{1}$ after removing those indices of $R$ with $\left.g\left(x^{(R)}\right)\right)=1$ encountered. Then we have $T_{k}^{\prime} \subset A_{1}^{\prime}$ and most likely, $C=A_{1} \backslash A_{1}^{\prime}$ has size $\Theta\left(n^{3 / 4}\right)$.

After the first stage, the algorithm has shrunk $A_{1}$ by $\Theta\left(n^{3 / 4}\right)$ while still making sure that variables of $T_{k}^{\prime}$ lie in $A_{1}^{\prime}$. In the second stage, the algorithm takes advantage of the smaller $A_{1}$ to search for $\ell$ in $A_{0}$, with each query essentially covering $\Theta\left(n^{3 / 4}\right)$ indices of $A_{0}$ :

Stage 2 . Randomly partition $A_{0}$ into $O\left(n^{1 / 4}\right)$ disjoint parts $A_{0,1}, \ldots$, each of size $|C|=\Theta\left(n^{3 / 4}\right)$. For each $A_{0, j}$, query $g\left(x^{\left(A_{0, j} \cup C\right)}\right)$. For each $A_{0, j}$ with $\ell \notin A_{0, j}, g$ must return 1 ; for the $A_{0, h}$ with $\ell \in A_{0, h}, g$ returns 0 with $\Omega(1)$ probability ${ }^{9}$ and when this happens, the algorithm finds a $O\left(n^{3 / 4}\right)$-size $A_{0, h} \subset A_{0}$ containing $\ell$. Let $y=x^{\left(A_{0, j} \cup C\right)}$.

Note that the algorithm cannot directly query $g\left(x^{\left(A_{0, j}\right)}\right)$ as the new string will be outside of the middle layers (unless $\left|A_{0, j}\right|=O(\sqrt{n})$, in which case one needs $\Omega(\sqrt{n})$ queries to cover $\left.A_{0}\right)$. This is only achieved by flipping $A_{0, j}$ and $C$ at the same time (in different directions) and this is the reason why we need the first stage to shrink $A_{1}$. In the last stage, the algorithm will find a violation for $y$, by providing $z<y$ with $g(z)=1$.

Stage 3. Randomly partition $A_{0, h}$ into $O\left(n^{1 / 4}\right)$ disjoint parts $\Delta_{1}, \ldots$, each of size $O(\sqrt{n})$. Query $g\left(y^{\left(\Delta_{i}\right)}\right)$. When $\ell \in \Delta_{i}, g\left(y^{\left(\Delta_{i}\right)}\right)=1$ with probability $\Omega(1)$, and $y^{\left(\Delta_{i}\right)} \prec y$.

\subsection{An Algorithm for Our Distributions}

The idea sketched above can be applied to our far from monotone distribution $\mathcal{D}_{\text {no }}$ from Section 1.3. It is slightly more complicated, since now the algorithm must attack two levels of Talagrand and

${ }^{9}$ Informally speaking, this is because the values of $g(x)$ and $g(y)$ essentially become independent when $x$ and $y$ are far from each other.

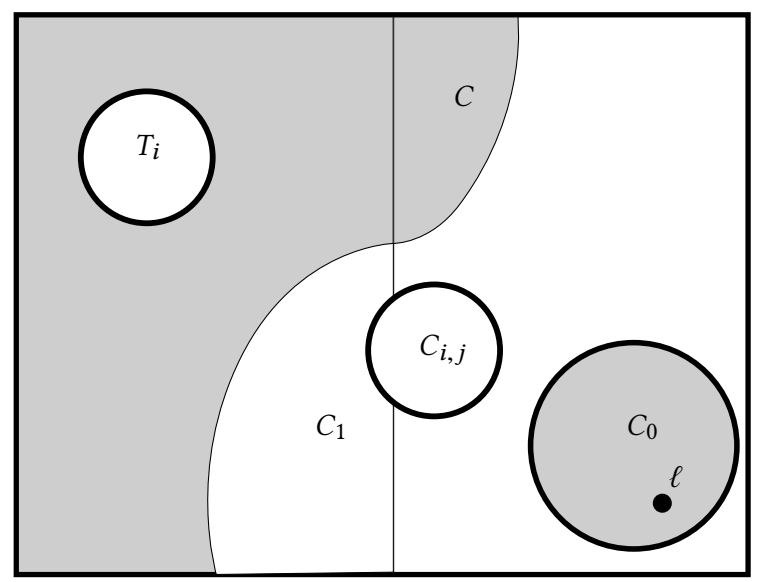

Figure 4: A visual representation of the algorithm for finding violations in the two-level Talagrand construction. The whole rectangle represents the set $[n]$, which is shaded for coordinates which are set to 1 , and clear for coordinates which are set to 0 . Here $T_{i}$ is the unique term satisfied and $C_{i, j}$ is the unique clause falsified. The functions $h_{i, j}$ is an anti-dictator with coordinate $\ell$. The sets illustrated represent the current knowledge at the end of Stage 3 of the algorithm. Note that $\left|C_{1}\right|=\Theta\left(n^{5 / 6}\right),|C|=\Theta\left(n^{2 / 3}\right),\left|C_{0}\right|=n^{5 / 6},\left|T_{i}\right|=\left|C_{i, j}\right|=\Theta(\sqrt{n})$.

will incur the query cost of $\tilde{O}\left(n^{1 / 3}\right)$ rather than $O\left(n^{1 / 4}\right)$. Similarly to Section 4.1 above, we will give a high level description, and not a formal analysis. The goal is to show the main obstacle one faces in improving the lower bound.

Assume $g$ is in the support of $\mathcal{D}_{\text {no. The algorithm works in stages }}$ and follows a similar pattern to the one described in Section 4.1 above. We may assume the algorithm has a string $x \in\{0,1\}^{n}$ where $x$ satisfies a unique term $T_{i}$, and falsifies no clauses, so $g(x)=1$ (this happens with $\Omega(1)$ probability for a random $x$ ).

Stage 1. Repeat the following for $n^{1 / 3}$ times: Pick a random subset $R \subset A_{1}$ of size $\sqrt{n}$ and query $g\left(x^{(R)}\right)$. Let $A_{1}^{\prime}$ denote $A_{1}$ after removing those indices of $R$ with $\left.g\left(x^{(R)}\right)\right)=1$ encountered. Then we have $T_{i} \subset A_{1}^{\prime}$ and most likely, $C_{1}=A_{1} \backslash A_{1}^{\prime}$ has size $\Theta\left(n^{5 / 6}\right)$.

The following stages will occur $n^{1 / 6}$ many times, and each makes $n^{1 / 6}$ many queries.

Stage 2. Pick a random subset $C_{0} \subset A_{0}$ of size $n^{5 / 6}$. Let $y=x^{\left(C_{1} \cup C_{0}\right)}$ and query $g(y)$. With probability $\Omega(1), g(y)$ satisfies the unique term $T_{i}($ as $\operatorname{did} x)$, falsifies a unique clause $C_{i, j}$, and $h_{i, j}(y)=0$. Additionally, with probability $\Omega\left(n^{-1 / 6}\right), h_{i, j}(y)=\overline{y_{\ell}}$, where $\ell \in C_{0}$.

Assume that $\ell \in C_{0}$, which happens with $\Omega\left(n^{-1 / 6}\right)$ probability. In the event this happens, we will likely find a violation.

Stage 3. Repeat the following for $n^{1 / 6}$ times: Pick a random subset $R \subset A_{0} \backslash C_{0}$ of size $\sqrt{n}$ and query $g\left(y^{(R)}\right)$. Let $A_{0}^{\prime}$ denote $A_{0} \backslash C_{0}$ after removing those indices of $R$ 
with $g\left(y^{(R)}\right)=0$. Let $C=\left(A_{0} \backslash C_{0}\right) \backslash A_{0}^{\prime}$, where very likely $|C|=\Theta\left(n^{2 / 3}\right)$. Our sets satisfy the following three conditions: 1) $T_{i} \subset A_{1}^{\prime}$; 2) $C_{i, j} \subset A_{0}^{\prime} \cup C_{1} \backslash C_{0}$; 3) $\ell \in C_{0}$. See Figure 4 for a visual representation of these sets.

Stage 4. Partition $C_{0}$ into $O\left(n^{1 / 6}\right)$ disjoint parts $C_{0,1}, \ldots$, each of size $\Theta\left(n^{2 / 3}\right)$ and query $g\left(y^{\left(C_{0, j} \cup C\right)}\right)$. For each $C_{0, j}$ with $\ell \notin C_{0, j}$ and no new terms are satisfied, $g$ must return 0 . If for some $C_{0, j}, g$ returns 1 , then either $\ell \in C_{0, j}$ and no new terms are satisfied, or new terms are satisfied; we can distinguish these cases with a statistical test.

The final stage is very similar to the final stage of Section 4.1. After Stage 4 , we assume that we have found a set $C_{0, j}$ containing $\ell$. We further partition $C_{0, j}$ (when $\left.g\left(y^{\left(C_{0, j} \cup C\right)}\right)=1\right)$ into $O\left(n^{1 / 6}\right)$ parts of size $\sqrt{n}$ each to find a violation. One can easily generalize the above algorithm sketch to $O(1)$-many levels of Talagrand. This suggests that the simple extension of our construction to $O(1)$ many levels (which still gives a far-from-monotone function) cannot achieve lower bounds better than $n^{1 / 3}$.

\section{DISCUSSION AND OPEN PROBLEMS}

While our two-level Talagrand functions for monotonicity testing looked promising at first sight, a few issues remain, which allow an algorithm to find a violating pair with $O\left(n^{1 / 3}\right)$ queries. However, for the problem of testing unateness, a different and much simpler pair of distributions allows us to overcome the $n^{1 / 3}$ obstacle for monotonicity and establish an $\widetilde{\Omega}(\sqrt{n})$ lower bound. The multiplexer maps for unateness turn out to be more resilient to the kinds of attacks sketched in Section 4, so one can imagine adapting them to the monotonicity setting. This leads us to the following conjecture:

CONJecture 5.1. Adaptivity does not help for monotonicity.

With regards to testing unateness, our adaptive lower bound of $\widetilde{\Omega}(\sqrt{n})$ exploited the existence of more resilient multiplexer maps. Although preliminary work suggests that the pair of distributions employed in our lower bound proof for unateness can be distinguished with $O(\sqrt{n})$ queries, it looks promising to us that small modifications to these distributions may yield lower bounds asymptotically higher than $\sqrt{n}$. This leads us to the following conjecture:

CONJECTuRE 5.2. Testing unateness is strictly harder than testing monotonicity.

\section{ACKNOWLEDGMENTS}

We thank Rocco Servedio and Li-Yang Tan for countless discussions and suggestions. This work is supported in part by NSF grants CCF1149257, CCF-1423100 and the NSF Graduate Research Fellowship under Grant No. DGE-16-44869.

\section{REFERENCES}

[1] Nir Ailon, Bernard Chazelle, Seshadhri Comandur, and Ding Liu. 2007. Estimating the distance to a monotone function. Random Structures and Algorithms 31, 3 (2007), 371-383.

[2] Roksana Baleshzar, Deeparnab Chakrabarty, Ramesh Krishnan S. Pallavoor, Sofya Raskhodnikova, and C. Seshadhri. 2017. Optimal Unateness Testers for RealValued Functions: Adaptivity Helps. arXiv preprint:1703.05199 (2017).

[3] Roksana Baleshzar, Meiram Murzabulatov, Ramesh Krishnan S. Pallavoor, and Sofya Raskhodnikova. 2016. Testing unateness of real-valued functions. arXiv preprint:1608.07652 (2016).
[4] Tugkan Batu, Ravi Kumar, and Ronitt Rubinfeld. 2004. Sublinear algorithms for testing monotone and unimodal distributions. In Proceedings of the 36th ACM Symposium on the Theory of Computing (STOC '2004). 381-390.

[5] Aleksandrs Belovs and Eric Blais. 2016. A polynomial lower bound for testing monotonicity. In Proceedings of the 48th ACM Symposium on the Theory of Computing (STOC '2016). 1021-1032.

[6] Eric Blais and Aleksandrs Belovs. 2015. Quantum algorithm for monotonicity testing on the hypercube. Theory of Computing 11, 16 (2015), 403-412.

[7] Eric Blais, Joshua Brody, and Kevin Matulef. 2012. Property testing lower bounds via communication complexity. Computational Complexity 21, 2 (2012), 311-358.

[8] Eric Blais, Sofya Raskhodnikova, and Grigory Yaroslavtsev. 2014. Lower bounds for testing properties of functions over hypergrid domains. In Proceedings of the 29th Conference on Computational Complexity (CCC '2014). 309-320.

[9] Jop Briët, Sourav Chakraborty, David García-Soriano, and Arie Matsliah. 2012. Monotonicity testing and shortest-path routing on the cube. Combinatorica 32, 1 (2012), 35-53.

[10] Deeparnab Chakrabarty and Seshadhri Comandur. 2016. An o(n) monotonicity tester for Boolean functions over the hypercube. SIAM f. Comput. 45, 2 (2016), 461-472.

[11] Deeparnab Chakrabarty and C. Seshadhri. 2013. Optimal bounds for monotonicity and Lipschitz testing over hypercubes and hypergrids. In Proceedings of the 45th ACM Symposium on the Theory of Computing (STOC '2013). 419-428.

[12] Deeparnab Chakrabarty and C. Seshadhri. 2014. An optimal lower bound for monotonicity testing over hypergrids. Theory of Computing 10, 17 (2014), 453464.

[13] Deeparnab Chakrabarty and C. Seshadhri. 2016. A $\widetilde{O}(n)$ non-adaptive tester for unateness. arXiv preprint:1608.06980 (2016).

[14] Xi Chen, Anindya De, Rocco A. Servedio, and Li-Yang Tan. 2015. Boolean function monotonicity testing requires (almost) $n^{1 / 2}$ non-adaptive queries. In Proceedings of the 47th ACM Symposium on the Theory of Computing (STOC '2015). 519-528.

[15] Xi Chen, Rocco A. Servedio, and Li-Yan Tan. 2014. New algorithms and lower bounds for monotonicity testing. In Proceedings of the 55th Annual IEEE Symposium on Foundations of Computer Science (FOCS '2014). 285-295.

[16] Xi Chen, Erik Waingarten, and Jinyu Xie. 2017. Beyond Talagrand functions: New lower bounds for testing monotonicity and unateness. arXiv preprint:1702.06997 (2017).

[17] Yevgeniy Dodis, Oded Goldreich, Eric Lehman, Sofya Raskhodnikova, Dana Ron, and Alex Samorodnitsky. 1999. Improved testing algorithms for monotonocity. In Approximation, Randomization and Combinatorial Optimization. Algorithms and Techniques.

[18] Funda Ergün, Sampath Kannan, S. Ravi Kumar, Ronitt Rubinfeld, and Mahesh Vishwanthan. 2000. Spot-checkers. F. Comput. System Sci. 60, 3 (2000), 717-751.

[19] Eldar Fischer. 2004. On the strength of comparisons in property testing. Information and Computation 189, 1 (2004), 107-116.

[20] Eldar Fischer, Eric Lehman, Ilan Newman, Sofya Raskhodnikova, Ronitt Rubinfeld, and Alex Samorodnitsky. 2002. Monotonicity testing over general poset domains. In Proceedings of the 34th ACM Symposium on the Theory of Computing (STOC '2002). 474-483.

[21] Oded Goldreich (Ed.). 2010. Property Testing: Current Research and Surveys. Vol. 6390. Springer-Verlag Berlin Heidelberg.

[22] Oded Goldreich, Shafi Goldwasser, Eric Lehman, and Dana Ron. 1998. Testing Monotonicity. In Proceedings of the 39th Annual IEEE Symposium on Foundations of Computer Science (FOCS '1998). 426-435.

[23] Oded Goldreich, Shafi Goldwasser, Eric Lehman, Dana Ron, and Alex Samordinsky. 2000. Testing Monotonicity. Combinatorica 20, 3 (2000), 301337.

[24] Shirley Halevy and Eyal Kushilevitz. 2008. Testing monotonicity over graph products. Random Structures and Algorithms 33, 1 (2008), 44-67.

[25] Subhash Khot, Dor Minzer, and Muli Safra. 2015. On monotonicity testing and boolean isoperimetric type theorems. In Proceedings of the 56th Annual IEEE Symposium on Foundations of Computer Science (FOCS '2015). 52-58.

[26] Subhash Khot and Igor Shinkar. 2016. An $\widetilde{O}(n)$ queries adaptive tester for unateness. In Approximation, Randomization and Combinatorial Optimization. Algorithms and Techniques. 37:1-37:7.

[27] Elchanan Mossel and Ryan O'Donnell. 2003. On the noise sensitivity of monotone functions. Random Structures and Algorithms 23, 3 (2003), 33-50.

[28] Dana Ron. 2008. Property testing: A learning theory perspective. Foundations and Trends ${ }^{\circledR}$ in Machine Learning 1, 3 (2008), 307-402.

[29] Dana Ron. 2010. Algorithmic and analysis techniques in property testing. Foundations and Trends ${ }^{\circledR}$ in Theoretical Computer Science 5, 2 (2010), 73-205.

[30] Dana Ron, Ronitt Rubinfeld, Muli Safra, Alex Samorodnitsky, and Omri Weinstein. 2012. Approximating the influence of monotone Boolean functions in $O(\sqrt{n})$ query complexity. ACM Transactions on Computation Theory 4, 4 (2012), 1-12.

[31] Ronitt Rubinfeld and Rocco A. Servedio. 2009. Testing monotone highdimensional distributions. Random Structures and Algorithms 34, 1 (2009), 24-44.

[32] Michel Talagrand. 1996. How much are increasing sets positively correlated? Combinatorica 16, 2 (1996), 243-258. 